\title{
A PROPERTY EQUIVALENT TO THE EXISTENCE OF SCALES
}

BY

\author{
HOWARD BECKER 1
}

\begin{abstract}
Let UNIF and SCALES be the propositions that every relation on $\mathbf{R}$ can be uniformized, and every subset of $\mathbf{R}$ admits a scale, respectively. For $A \subset \mathbf{R}$, let $w(A)$ denote the Wadge ordinal of $A$, and let $\delta_{1}^{1}(A)$ be the supremum of the ordinals realized in the pointclass $\Delta_{1}^{1}(A)$.

THEOREM (AD). The following are equivalent:

(a) SCALES,

(b) UNIF + the set $\left\{w(A): \delta_{1}^{1}(A)=(w(A))^{+}\right\}$contains an $\omega$-cub subset of $\Theta$.

Using this theorem, Woodin has shown that if the theory $(\mathrm{ZF}+\mathrm{DC}+\mathrm{AD}+$ UNIF) is consistent, then the theory (ZF + DC $+\mathrm{AD}_{\mathbf{R}}+$ SCALES) is also consistent. In this paper we give a proof of the above theorem and of a local version of it. We also study the ordinal $\delta_{1}^{1}(A)$ and give several characterizations of it.
\end{abstract}

This paper is a contribution to descriptive set theory, the study of definable sets of reals, under the assumption of the axiom of determinacy (AD). Specifically, our aim is to study some properties which are (locally or globally) equivalent to the existence of scales on pointsets. In $\$ 1$ we give a brief introduction to this general topic, and in \$2 we will state our equivalence results. We work in $\mathrm{ZF}+\mathrm{DC}$ and work with the space ${ }^{\omega} \omega(=\mathbf{R}=$ the reals $)$. Our basic reference is Moschovakis [12].

1. Determinacy, uniformization, and scales. $A D$ is the proposition that every infinite two-person game of perfect information on $\omega$ is determined. AD contradicts the axiom of choice (AC), but is probably consistent with $\mathrm{ZF}+\mathrm{DC}$. While AC implies there are nondetermined games, it is a quite reasonable hypothesis that every definable game is determined. And if it is true (in $V$ ) that every game that is ordinal definable from a real is determined, then $L[R] \vDash(\mathrm{ZF}+\mathrm{DC}+\mathrm{AD})$. Thus $L[\mathbf{R}]$ is a natural model of $\mathrm{AD}$, and theorems proved from this axiom are often thought of as being theorems about $L[\mathbf{R}]$. But $L[\mathbf{R}]$ is not necessarily the only natural model for AD. There may be larger models, for example $L\left[\mathbf{R}, \mathbf{R}^{\sharp}\right]$, where $\mathbf{R}^{\sharp} \subset \mathbf{R}$ is " the sharp" of $L[\mathbf{R}]$, or the Chang-Kunen model $[2,7]$, that is, the smallest model $M$ of ZF such that $M$ contains all ordinals and ${ }^{\omega} M \subset M$.

The global equivalence mentioned above can best be thought of as a statement about axioms stronger than AD. We discuss several such axioms here. One such axiom is $\mathrm{AD}_{\mathbf{R}}$, the proposition that all games on reals are determined. Mycielski [13] showed that $\mathrm{AD}_{\omega_{1}}$ and $\mathrm{AD}_{\text {Power(R) }}$ are inconsistent (with $\mathrm{ZF}+\mathrm{DC}$ ), hence $\mathrm{AD}_{\mathbf{R}}$

Received by the editors February 28, 1983 and, in revised form, March 12, 1984.

1980 Mathematics Subject Classification. Primary 03E60.

${ }^{1}$ Partially supported by NSF Grant MCS82-11328. 
seems to be the only possible consistent strengthening of $\mathrm{AD}$ of this form (recall that under $\mathrm{AD}, \omega_{1} \nless 2^{\omega}$ ).

Let UNIF be the proposition that every binary relation on the reals can be uniformized (for definitions, see [12]). UNIF is, of course, a weak form of AC. UNIF is also essentially a determinacy axiom (a weak form of $\mathrm{AD}_{\mathbf{R}}$ ), since it is easy to see that UNIF is equivalent to the determinacy of all games on $\mathbf{R}$ in which each player makes only one move, and also equivalent to the determinacy of all games in which player I makes one move, choosing a real, and then player II makes $\omega$ integer moves. Thus $\mathrm{AD}+\mathrm{UNIF}$ is the weakest determinacy axiom extending AD. This axiom is false in $L[\mathbf{R}]$, or in $L[\mathbf{R}, A]$ for any $A \subset \mathbf{R}$, since if $L[\mathbf{R}, A] \vDash \mathrm{AD}$, then in $L[\mathbf{R}, A]$ the following relation $P$ on $\mathbf{R}$ cannot be uniformized:

$$
P(x, y) \Leftrightarrow(y \text { is not ordinal definable from } A \text { and } x) \text {. }
$$

If $L[\mathbf{R}]$ is not a model, what is? We do not yet have a completely satisfactory answer to this question, although some recent work of Woodin provides a partial answer. In fact there is no known proof of the consistency of $(\mathrm{ZF}+\mathrm{DC}+\mathrm{AD}+$ UNIF) from any axiom that could conceivably be true in $V$, that is to say, there is no known analog of the theorem of ZFC that if every definable game is determined then $L[R] \vDash A D$. The search for a model and an analogous theorem for (AD + UNIF) or $A D_{\mathbf{R}}$ is one of the major open problems in the field. It has been suggested (Solovay, Blass [1]) that $\mathrm{AD}_{\mathbf{R}}$ may hold in the Chang-Kunen model.

Let SCALES be the proposition that every set of reals admits a scale, that is, is $\kappa$-Suslin for some $\kappa$. (Trivially, AC $\Rightarrow$ SCALES, but those scales are uninteresting. We are here concerned only with the choiceless universe of $A D$.) If a relation admits a scale it can be uniformized. Indeed the concept of scale was originally introduced by Moschovakis [11] in order to prove uniformization theorems (e.g. $\Pi_{3}^{1}$ relations have $\Pi_{3}^{1}$ uniformizations). It has subsequently been shown that scales have numerous applications besides uniformization, some of which will be considered in this paper. Woodin (unpublished) proved that the existence of scales implies $\mathrm{AD}_{\mathbf{R}}$. Hence we have:

1.1. $(\mathrm{AD}+\mathrm{SCALES}) \Rightarrow \mathrm{AD}_{\mathbf{R}} \Rightarrow(\mathrm{AD}+\mathrm{UNIF})$.

$\mathrm{AD}+\mathrm{SCALES}$ is the strongest axiom that will be considered here. (The only stronger axioms of the sort usually considered by mathematicians who work with $\mathrm{AD}$ are obtained by adding to AD + SCALES an assertion that $\Theta$ is "large", for example, that $\Theta$ is regular-cf. Solovay [14].)

For both of the implications in 1.1, the converse is still an open question. We conjecture that AD + UNIF implies SCALES, and hence all three axioms are equivalent. If true, this is of interest for several reasons. It shows that there is basically only one way to strengthen $\mathrm{AD}$, and it may also be helpful in the search for a model of $\mathrm{AD}_{\mathbf{R}}$. But its main interest arises from the fact that $\mathrm{AD}+\mathrm{SCALES}$ has many consequences that are not known to be consequences of $\operatorname{AD}_{\mathbf{R}} \cdot\left(\mathrm{AD}_{\mathbf{R}}\right.$ is the officially accepted axiom and SCALES is not.) Two examples of such consequences of AD + SCALES that are relevant to this paper are:

1.2. (1) Every set of reals is $\infty$-Borel, that is, is a member of the smallest family of sets containing the open sets and closed under complements and well-ordered unions 
[12, 2E.2], and in fact is effectively $\infty$-Borel in the sense of [12, p. 538], i.e., there is a well-founded tree on ordinals which tells how to construct the set via complements and well-ordered unions.

(2) There are arbitrarily large (in $\Theta$ ) regular successor cardinals (see 2.5 below).

This paper can be regarded as being about weak versions of the (as yet unproved) proposition that $(A D+U N I F) \Rightarrow$ SCALES.

2. A property equivalent to the existence of scales. Next we consider a local property of pointsets and pointclasses, the Kunen-Martin property, which involves the existence of upper bounds on the ranks of well-founded relations. We study it in an abstract setting. The framework for the study of arbitrary pointclasses is the concept of Wadge degrees with which we assume the reader is familiar; Van Wesep [16] is a good reference. For $A \subset \mathbf{R}$, let $w(A)$ denote the Wadge ordinal of $A$. Since this makes no sense without $\mathrm{AD}$, for the rest of this paper $\mathrm{AD}$ is always assumed.

For any $A \subset \mathbf{R}$ the pointclass $\Pi_{1}^{1}(A)$ is the smallest class containing $A$ and closed under \&, $\vee, \exists^{\omega}, \forall^{\omega}, \forall^{\mathbf{R}}$, and continuous substitution. This class always exists and is R-parametrized [10]. Let $\Sigma_{1}^{1}(A)$ be the dual class of $\Pi_{1}^{1}(A)$ and let $\Delta_{1}^{1}(A)$ be $\Pi_{1}^{1}(A) \cap \Sigma_{1}^{1}(A)$. For any $A \subset \mathbf{R}$ let

$$
\delta_{1}^{1}(A)=\sup \left\{\operatorname{rank}(\prec): \prec \text { a strict well-founded relation on } \mathbf{R} \& \prec \in \Delta_{1}^{1}(A)\right\} \text {. }
$$

Thus we have assigned to each set $A \subset \mathbf{R}$ two ordinals, $w(A)$ and $\delta_{1}^{1}(A)$ (both of which clearly depend only on the Wadge degree of $A$ ). A set $A \subset \mathbf{R}$ has the Kunen-Martin property if $\delta_{1}^{1}(A) \leqslant(w(A))^{+}$.

\subsection{TheOREM (AD). If $A$ is $w(A)$-Suslin then $\delta_{1}^{1}(A) \leqslant(w(A))^{+}$.}

Proof. By the closure properties of the class of $w(A)$-Suslin sets, every $\Sigma_{1}^{1}(A)$ relation is $w(A)$-Suslin; hence by the Kunen-Martin Theorem [12, 2G.2], $\delta_{1}^{1}(A) \leqslant$ $(w(A))^{+}$.

The main theorem to be proved in this paper is essentially a converse of 2.1: for certain sets $A$, the Kunen-Martin property for $A$ implies that $A$ is $w(A)$-Suslin (and hence that $A$ admits a scale).

2.2. Definition. Let $A \subset \mathbf{R}$ and let $\Lambda=\{B: w(B)<w(A)\}$. $A$ is useful if:

(a) $w(A)$ is a limit ordinal and $\operatorname{cof}(w(A))=\omega$.

(b) For all $B \in \Lambda, \operatorname{Env}\left(B,{ }^{3} E\right) \subset \Lambda$.

(c) Any relation in $\Lambda$ can be uniformized by a relation in $\Lambda$.

See Moschovakis [9] for results about envelopes. All that we use here is that $\operatorname{Env}\left(B,{ }^{3} E\right)$ is a Spector pointclass $\Gamma$ containing $B$ and $\neg B$, closed under $\forall^{\mathbf{R}}$, and with the property that $\Delta(=\Gamma \cap \breve{\Gamma})$ is (uniformly) closed under $\forall^{\mathbf{R}}$. Such a class $\Gamma$ always exists and in $\operatorname{fact} \operatorname{Env}\left(B,{ }^{3} E\right)$ is the smallest such class. The reader who prefers inductive definability to higher-type recursion can substitute the pointclass $\operatorname{IND}(B)$ of $[12, \S 7 C]$ for $\operatorname{Env}\left(B,{ }^{3} E\right)$ in the above definition; this will make the main theorem (Theorem 2.3) weaker. Notice that UNIF implies that $\{w(A): A$ useful $\}$ contains an $\omega$-closed unbounded ( $\omega$-cub) subset of $\Theta$. Similarly if $\Delta$ has a lot of closure properties and the uniformization property (e.g. $\left.\Delta=\left(\Delta_{1}^{2}\right)^{L[R]}[8]\right)$, then 
$\{w(A): A$ useful $\}$ contains an $\omega$-cub subset of $\delta$. So a lot of sets (in some sense almost all sets) are useful.

2.3. Main Theorem (AD). Let $A$ be useful. If $\delta_{1}^{1}(A) \leqslant(w(A))^{+}$, then $A$ is $w(A)$-Suslin.

This theorem differs from previous theorems on the existence of scales in a significant way. The previous theorems (such as Moschovakis [12, 6C] or MartinMoschovakis-Steel [8]) all assert that the class of sets which admit scales has certain closure properties; 2.3 is not such an assertion.

If $A$ is useful then the closure properties of $\Lambda$ imply that $\delta_{1}^{1}(A) \geqslant(w(A))^{+}$(see Lemma 2.3.1 of [6]). This fact, plus 2.1 and 2.3, gives

2.4. Corollary (AD). Let $A$ be useful. The following are equivalent:

(a) $A$ is $w(A)$-Suslin.

(b) $\delta_{1}^{1}(A)=(w(A))^{+}$.

This corollary is the local equivalence mentioned at the beginning of the paper-for useful $A$, it is a necessary and sufficient condition for $A$ to be $w(A)$-Suslin. Clearly SCALES implies that the set $\{w(A): A$ is $w(A)$-Suslin $\}$ contains an $\omega$-cub subset of $\Theta$. This fact, together with 2.4 , gives us a global equivalence.

2.5. COROLLARY (AD). The following are equivalent:

(a) SCALES.

(b) UNIF + the set of ordinals $\left\{w(A): \delta_{1}^{1}(A)=(w(A))^{+}\right\}$contains an $\omega$-cub subset of $\Theta$.

Since $\delta_{1}^{1}(A)$ is always regular [12,7D.11], this justifies $1.2(2)$. The author conjectures that it is provable (from AD alone) that for useful $A, \delta_{1}^{1}(A)=(w(A))^{+}$. We will have more to say about this conjecture in $\$ 7$. If true, the above results show that this conjecture implies our previous conjecture that UNIF $\Rightarrow$ SCALES. Even more evidence for UNIF $\Rightarrow$ SCALES is provided by some (unpublished) work of Woodin, which we now describe.

Assume AD + UNIF, and let $\mathscr{P} \subset$ Power(R) be some set closed under continuous preimages, that is, $\mathscr{P}$ is an initial segment of the Wadge degrees. For any such $\mathscr{P}$, $L[\mathbf{R}, \mathscr{P}]$ will be a model of $\mathrm{ZF}+\mathrm{AD}$. Call $\mathscr{P}$ nice if:

(1) $($ Power $(\mathbf{R}) \cap L[\mathbf{R}, \mathscr{P}])=\mathscr{P}$,

(2) $L[\mathbf{R}, \mathscr{P}] \vDash \mathrm{DC}$,

(3) $L[\mathbf{R}, \mathscr{P}] \vDash U N I F$.

There is a nice $\mathscr{P}$, namely $\mathscr{P}=$ Power $(\mathbf{R})$, and there may be others. Since the Wadge degrees are well ordered, there is a minimal nice $\mathscr{P}$. Let $M=L[\mathbf{R}, \mathscr{P}]$ where $\mathscr{P}$ is minimal nice. Woodin has proved that

$M \vDash$ Every set of reals is effectively $\infty$-Borel.

(See 1.2(1) for definition. See Solovay [14] for more on this type of model.)

After Corollary 2.5 was proved, he announced the following

2.6. THEOREM (WOODIN; $\mathrm{AD}+\mathrm{UNIF).} \mathrm{If} \mathrm{every} \mathrm{set} \mathrm{of} \mathrm{reals} \mathrm{is} \mathrm{effectively} \infty$-Borel, then the set $\left\{w(A): \delta_{1}^{1}(A)=(w(A))^{+}\right\}$contains an $\omega$-cub subset of $\Theta$. 
Hence the conclusion of 2.6 holds in $M$, and so by $2.5, M \vDash$ SCALES. This shows that if $(\mathrm{ZF}+\mathrm{DC}+\mathrm{AD}+\mathrm{UNIF})$ is consistent, then $\left(\mathrm{ZF}+\mathrm{DC}+\mathrm{AD}_{\mathbf{R}}+\right.$ SCALES) is also consistent. (By Solovay [14] these theories have much greater consistency strength than (ZF + DC + AD) does.) Woodin's work will be published elsewhere.

$\S \S 3-6$ contain a proof of the Main Theorem (2.3). The basic outline of the proof is contained in $\S 3$, where the Main Theorem is reduced to one technical fact: that a particular integer-real game is determined and one player has a simple winning strategy. This technical fact is proved in $\$ \$ 4-6$. The proof is a modification of the method of Harrington-Kechris [3]. The techniques of $\$ \S 4-6$ are of some interest in themselves and have applications beyond the one in this paper. For example, Kechris [5] has used this technique to study the complexity of measures, e.g. he proved that every measure on $\delta_{3}^{1}$ is $\Delta_{5}^{1}$-in-the-codes.

In $\$ 7$ we study the ordinal $\delta_{1}^{1}(A)$ for sets $A$ such that $\operatorname{cof}(w(A))=\omega$ and $\Lambda$ is strongly-closed; this includes all the useful sets. We give a number of different characterizations of the ordinal. These characterizations are proved from AD alone and have nothing to do with either uniformization or scales. But assuming $A$ is useful, we get a new equivalence in 2.4 , and assuming UNIF in 2.5 , that is, we get new examples of properties equivalent to the existence of scales. $\$ 7$ can be read independently of $\$ \$ 3-6$.

3. Proof of the main theorem. Fix a useful set $A$ such that $\delta_{1}^{1}(A)=(w(A))^{+}$. Let $\Lambda=\{B: w(B)<w(A)\}$.

Suppose $\Gamma \subset \Lambda$ is an $\omega$-parametrized (lightface) pointclass and $G \subset \mathbf{R} \times \mathbf{R}^{2}$ is in $\Gamma$ and universal for $\Gamma$ subsets of $\mathbf{R}^{2}$. By definition of useful (2.2), there is a $G^{*} \subset \mathbf{R}^{2} \times \mathbf{R}$ such that $G^{*}$ is in $\Lambda$ and $G^{*}$ uniformizes $G$ (considered as a subset of $\left.\mathbf{R}^{2} \times \mathbf{R}\right)$, that is, $G^{*} \subset G$ and

$$
\forall x, y\left[\exists z G(x, y, z) \Leftrightarrow \exists ! z G^{*}(x, y, z)\right] .
$$

Now if $\Gamma^{\prime}$ is any $\omega$-parametrized pointclass containing $G^{*}$, then since $G$ is universal, for all $x \in \mathbf{R}$, every $\Gamma(x)$ relation has a $\Gamma^{\prime}(x)$ uniformization; moreover this is uniform, i.e. there is a recursive function taking a $\Gamma(x)$-index (w.r.t. $G$ ) for a $\Gamma(x)$ relation $R$ to a $\Gamma^{\prime}(x)$-index (w.r.t. any universal set) for a $\Gamma^{\prime}(x)$ relation $R^{*}$ such that $R^{*}$ uniformizes $R$. Using this remark, the next lemma is easy to prove.

3.1. LEMMA. There exists a sequence of ordinals $\kappa_{0}, \dot{\kappa}_{1}, \kappa_{2}, \ldots$, a sequence of prewellorderings $\leqslant_{0}, \leqslant_{1}, \leqslant_{2}, \ldots$, and two sequences of pointclasses $\Gamma_{0}^{\mathrm{I}}, \Gamma_{1}^{\mathrm{I}}, \Gamma_{2}^{\mathrm{I}}, \ldots$ and $\Gamma_{0}^{\mathrm{II}}, \Gamma_{1}^{\mathrm{II}}, \Gamma_{2}^{\mathrm{II}} \ldots$ such that:

(1) $\kappa_{0}<\kappa_{1}<\kappa_{2}<\cdots$.

(2) $w(A)=\sup \kappa_{i}$.

(3) For all $i, \leqslant_{i}$ is a prewellordering of $\mathbf{R}$ of order type $\kappa_{i}$.

(4) $\Gamma_{0}^{\mathrm{I}} \subset \Gamma_{0}^{\mathrm{II}} \subset \Gamma_{1}^{\mathrm{I}} \subset \Gamma_{1}^{\mathrm{II}} \subset \Gamma_{2}^{\mathrm{I}} \subset \Gamma_{2}^{\mathrm{II}} \subset \cdots$.

(5) For all $i, \Gamma_{i}^{\mathrm{I}}$ and $\Gamma_{i}^{\mathrm{II}}$ are both Spector pointclasses closed under $\forall^{\mathbf{R}}$ and with the property that $\Delta_{i}^{\mathrm{I}}$ and $\Delta_{i}^{\mathrm{II}}$ are both (uniformly) closed under $\forall^{\mathbf{R}}$.

(6) For all $i, \Gamma_{i}^{\mathrm{I}} \subset \Lambda$. 
(7) For all $i, \leqslant_{i} \in \Delta_{i}^{\mathrm{II}}$.

(8) For all $i$, for all $x \in \mathbf{R}$, every $\Gamma_{i}^{\mathrm{I}}(x)$ relation has a $\Delta_{i}^{\mathrm{II}}(x)$ uniformization (uniformly).

(9) For all $i$, for all $x \in \mathbf{R}$, every $\Gamma_{i}^{\mathrm{II}}(x)$ relation has a $\Delta_{i+1}^{\mathrm{I}}(x)$ uniformization (uniformly).

Fix $\kappa_{i}$ 's, $\leqslant{ }_{i}$ 's, $\Gamma_{i}^{\mathrm{I}}$ 's and $\Gamma_{i}^{\mathrm{II}}$ 's satisfying Lemma 3.1.

3.2. Definition. A $\bar{\kappa}$-tree is a tree $T$ on $w(A)$ such that if $\left(\xi_{0}, \xi_{1}, \ldots, \xi_{n}\right) \in T$, then $\xi_{0}<\kappa_{0} \& \xi_{1}<\kappa_{1} \& \cdots \& \xi_{n}<\kappa_{n}$.

3.3. LeMmA. For all $\eta<(w(A))^{+}$, there exists a $\bar{\kappa}$-tree $T$ such that $T$ is well founded and $\operatorname{rank}(T)>\eta$.

By the Moschovakis coding lemma [10], any subset of $\left(\kappa_{0} \times \kappa_{1} \times \cdots \times \kappa_{n}\right)$ is $\Sigma_{1}^{1}\left(\leqslant_{0}, \leqslant_{1}, \ldots, \leqslant_{n}\right)$-in-the-codes, where coordinate $i$ is coded with respect to $\leqslant_{i}$. Code relations on $\kappa_{0} \times \cdots \times \kappa_{n}$ via some fixed universal $\Sigma_{1}^{1}\left(\leqslant_{0}, \ldots, \leqslant_{n}\right)$ set. Let $P^{n}(x)$ denote the subset of $\kappa_{0} \times \cdots \times \kappa_{n}$ encoded by $x$. It will be convenient to have every real encode some subset of $\kappa_{0} \times \cdots \times \kappa_{n}$. Notice that by the closure properties of $\Delta_{n}^{\mathrm{II}}$, this coding of $\operatorname{Power}\left(\kappa_{0} \times \cdots \times \kappa_{n}\right)$ is $\Delta_{n}^{\mathrm{II}}$, that is, the following $(n+2)$-ary relation $E$ is $\Delta_{n}^{\mathrm{II}}$ :

3.4. $E\left(x, y_{0}, \ldots, y_{n}\right) \Leftrightarrow\left[(\forall i \leqslant n)\left(y_{i}\right.\right.$ encodes (w.r.t. $\left.\leqslant_{i}\right)$ the ordinal $\left.\xi_{i}<\kappa_{i}\right)$

$$
\left.\&\left(\xi_{0}, \ldots, \xi_{n}\right) \in P^{n}(x)\right] .
$$

We next encode $\bar{\kappa}$-trees by elements of ${ }^{\omega} \mathbf{R}$ (which are essentially reals). Let $\operatorname{Tr} \subset{ }^{\omega} \mathbf{R}$ be as follows:

3.5. $\operatorname{Tr}\left(\left\langle x_{0}, x_{1}, \ldots\right\rangle\right) \Leftrightarrow \forall n\left(P^{n+1}\left(x_{n+1}\right)\right.$ extends $\left.P^{n}\left(x_{n}\right)\right)$.

Saying $P^{n+1}\left(x_{n+1}\right)$ extends $P^{n}\left(x_{n}\right)$ means that for any length $n+1$ sequence $u \in \kappa_{0} \times \cdots \times \kappa_{n}$, and for any $\xi<\kappa_{n+1}$, if $u^{\frown}(\xi) \in P^{n+1}\left(x_{n+1}\right)$ then $u \in P^{n}\left(x_{n}\right)$. $\operatorname{Tr}$ is the set of codes for $\bar{\kappa}$-trees. If $\bar{x}=\left\langle x_{0}, x_{1}, \ldots\right\rangle \in \mathbf{T r}$, then $\bar{x}$ encodes the $\bar{\kappa}$-tree $\operatorname{Tr}_{\bar{x}}=\bigcup_{n \in \omega} P^{n}\left(x_{n}\right)$. And every $\bar{\kappa}$-tree has a code.

Consider the following game $\mathscr{G}$. On move $i$, I plays an integer $y(i)$ and II plays a real $x_{i}$. Thus I produces $y \in{ }^{\omega} \omega=\mathbf{R}$ and II produces $\bar{x}=\left\langle x_{0}, x_{1}, x_{2}, \ldots\right\rangle \in{ }^{\omega} \mathbf{R}$.

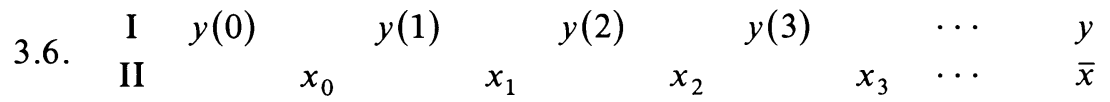

3.7. II wins $\Leftrightarrow\left[\bar{x} \in \operatorname{Tr} \&\left[\left(y \in A \& \operatorname{Tr}_{\bar{x}}\right.\right.\right.$ is not well founded $)$

$$
\text { or } \left.\left.\left(y \notin A \& \operatorname{Tr}_{\bar{x}} \text { is well founded }\right)\right]\right] \text {. }
$$

In the game $\mathscr{G}, \mathrm{II}$ is essentially playing a tree in $\omega$ moves - on move $n$, II plays the set of length $n$ sequences of the tree. The closed condition $\bar{x} \in \operatorname{Tr}$ ensures that II actually does play a tree (or else loses at some finite time). The game $\mathscr{G}$ is similar to a Wadge game; in a sense, II is trying to "Wadge-reduce" the set $A$ to the set of non-well-founded trees.

3.8. LEMMA. If II has a winning strategy for $\mathscr{G}$, then $A$ is $w(A)$-Suslin. 
Proof. Let $\tau$ be a winning strategy for II for $\mathscr{G}$. Let

$$
\begin{aligned}
T=\left\{\left(a_{0}, \xi_{0}, a_{1}, \xi_{1}, \ldots, a_{n}, \xi_{n}\right) \in(\omega \times w(A))^{n+1}:\right. \\
\xi_{0} \in P^{0}\left(\tau\left(a_{0}\right)\right) \&\left(\xi_{0}, \xi_{1}\right) \in P^{1}\left(\tau\left(a_{0}, a_{1}\right)\right) \\
\left.\& \cdots \&\left(\xi_{0}, \ldots, \xi_{n}\right) \in P^{n}\left(\tau\left(a_{0}, \ldots, a_{n}\right)\right)\right\} .
\end{aligned}
$$

Clearly $T$ is a tree on $\omega \times w(A)$ (in fact a $\bar{\kappa}$-tree). And by definition of the payoff set for II, $p[T]=A$.

The converse of 3.8 is also true. This type of game was invented by Steel. (For any $A^{\prime} \subset \mathbf{R}$ and any cardinal $\kappa^{\prime}<\Theta$, a similar game $\mathscr{G}\left(A^{\prime}, \kappa^{\prime}\right)$ can be defined. Steel showed, as above, that II has a winning strategy for $\mathscr{G}\left(A^{\prime}, \kappa^{\prime}\right)$ iff $A^{\prime}$ is $\kappa^{\prime}$-Suslin.)

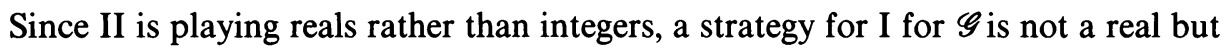
a higher type object, essentially a set of reals. If $\sigma$ is a strategy for I for $\mathscr{G}$, let $\sigma_{n}$ : $\mathbf{R}^{n} \rightarrow \omega$ be the piece of the strategy $\sigma$ which tells I how to play on move $n+1$; thus $\sigma$ is $\sigma_{0} \cup \sigma_{1} \cup \sigma_{2} \cup \cdots$. A strategy $\sigma$ for I for $\mathscr{G}$ is simple if for all $n \in \omega, \sigma_{n} \in \Lambda$. (Since $\Lambda$ is not closed under countable unions, a simple strategy is not necessarily in

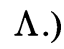

\subsection{LEMMA. I does not have a simple winning strategy for $\mathscr{G}$.}

Proof. Suppose $\sigma$ is a winning strategy for $I$ for $\mathscr{G}$. Let $Y \subset\left(\mathbf{R} \times{ }^{\omega} \mathbf{R} \times(w(A))^{<\omega}\right)$ be as follows:

$$
\begin{aligned}
& \left\{\left(y, \bar{x},\left\langle\xi_{0}, \xi_{1}, \ldots, \xi_{n}\right\rangle\right): y \in A \&(y, \bar{x})\right. \text { is consistent with } \\
& \text { the strategy } \left.\sigma \& \bar{x} \in \operatorname{Tr} \&\left\langle\xi_{0}, \xi_{1}, \ldots, \xi_{n}\right\rangle \in \operatorname{Tr}_{\bar{x}}\right\} .
\end{aligned}
$$

Let $\triangleleft$ be the following binary relation on $Y$ :

$$
(y, \bar{x}, \bar{\xi}) \triangleleft\left(y^{\prime}, \bar{x}^{\prime}, \bar{\xi}^{\prime}\right) \Leftrightarrow\left(y=y^{\prime} \& \bar{x}=\bar{x}^{\prime} \& \bar{\xi}^{\prime} \prec \bar{\xi}\right) .
$$

Since $\sigma$ is winning, if $(y, \bar{x}, \bar{\xi}) \in Y$ then $\operatorname{Tr}_{\bar{x}}$ is a well-founded tree. Therefore the relation $\triangleleft$ is (strictly) well founded, and by 3.3 it must have rank $(w(A))^{+}$. For $z \in \mathbf{R}$, let $|z|_{i}$ be the ordinal $\left(<\kappa_{i}\right)$ which is $z$ 's rank in the prewellordering $\leqslant_{i}$. Let $Y^{*} \subset \mathbf{R}^{3}$ be the code set of $Y$, that is,

$$
\begin{array}{r}
Y^{*}=\left\{(y, x, z) \in \mathbf{R}^{3}: z=\left\langle z_{0}, \ldots, z_{n}\right\rangle \&\left(y,\left\langle(x)_{0},(x)_{1},(x)_{2}, \ldots\right\rangle,\right.\right. \\
\left.\left.\left\langle\left|z_{0}\right|_{0},\left|z_{1}\right|_{1}, \ldots,\left|z_{n}\right|_{n}\right\rangle\right) \in Y\right\} .
\end{array}
$$

Let $\triangleleft^{*}$ be the code set of $\triangleleft$. Then $\triangleleft^{*}$ is a strict well-founded relation on $Y^{*}$ of rank $(w(A))^{+}$.

If $\sigma$ is simple, then some routine quantifier-counting shows that $Y^{*}$ and $\triangleleft^{*}$ are both $\Delta_{1}^{1}(A)$. Hence there is a $\Delta_{1}^{1}(A)$ well-founded relation on $\mathbf{R}$ of rank $(w(A))^{+}$, contrary to the hypothesis of the main theorem.

In light of Lemmas 3.8 and 3.9, to complete the proof of the Main Theorem it will suffice to show the following:

3.10. Either I has a simple winning strategy for $\mathscr{G}$ or II has a winning strategy for $\mathscr{G}$.

This will be done in three steps. In $\S 4$ we will define a game $\mathscr{G}^{*}$ on $\omega$ (which by $\mathrm{AD}$ is determined). After defining $\mathscr{G}^{*}$, in $\$ \S 5$ and 6 we will prove the following two lemmas, and thereby complete the proof of the Main Theorem. 
3.11. LEMMA A. If I has a winning strategy for $\mathscr{G}^{*}$, then I has a simple winning strategy for $\mathscr{G}$.

3.12. LEMMA B. If II has a winning strategy for $\mathscr{G}^{*}$, then II has a winning strategy for $\mathscr{G}$.

It is true that $\mathrm{AD}+\mathrm{UNIF}$ implies that every integer-real game is determined (Kechris, unpublished). But in 3.10 the determinacy of the game $\mathscr{G}$ is not the real issue. The claim being made in 3.10 is that if I has a winning strategy, then he has one which is simple. In general, the complexity of the winning strategy for the player playing integers depends on the complexity of the game. To say a tree $\operatorname{Tr}_{\bar{x}}$ is well founded is $\Pi_{1}^{1}(A)$, so the payoff set for the game $\mathscr{G}(3.7)$ is a difference of $\Pi_{1}^{1}(A)$ sets, yet in 3.10 we are demanding a $\Delta_{1}^{1}(A)$ strategy, i.e. we need a strategy of lower Wadge degree than the game. It is not true that for arbitrary integer-real games which are differences of $\Pi_{1}^{1}(A)$ sets, there is a $\Delta_{1}^{1}(A)$ winning strategy-there is something special about this particular game $\mathscr{G}$ that will come up in the proof. We have one more remark on the proof: in proving the Main Theorem we have not yet used uniformization; this will come up in the proof of 3.10 .

4. Definition of the game $\mathscr{G}^{*}$. $\mathscr{G}^{*}$ will be a game on $\omega$ which simulates the integer-real game $\mathscr{G}$. Harrington and Kechris [3] showed how to simulate a certain type of game on $\mathbf{R}$ (namely a coded ordinal game) by a game on $\omega$, and thus prove not only the determinacy of the original game but also the existence of a "definable" winning strategy. The simulation to be described here is a modified form of the basic Harrington-Kechris method. (The reader need not be familar with [3] to follow our presentation-we will redo everything here.)

In $\mathscr{G}^{*}$ it will be convenient to work with the space $\mathscr{C}={ }^{\omega} 2$ (Cantor space), rather than with R. Recall that we have fixed pointclasses $\Gamma_{n}^{\mathrm{I}}, \Gamma_{n}^{\mathrm{II}}$ satisfying Lemma 3.1. For each such pointclass, fix a universal set which is good, i.e. which satisfies the $S-m-n$ theorem $[12,3 \mathrm{H}]$. Phrases such as "the $\Gamma_{n}^{\mathrm{I}}(b)$ subset of $\omega^{2}$ with index $e$ " implicitly refer to these fixed universal sets. A formal definition of the game $\mathscr{G}^{*}$ follows.

For $n \in \omega, a$ is a $(\mathrm{I}, n)$-precode if $a=\langle e, b\rangle, e \in \omega, b \in \mathscr{C}$, the $\Gamma_{n}^{\mathrm{I}}(b)$ subset of $\omega^{2}$ with index $e$ is a total function and that function encodes a perfect binary tree $T_{a} \subset 2^{<\omega}$. Here $\langle e, b\rangle$ denotes the following element of $\mathscr{C}$ :

$$
(\underbrace{0,0,0, \ldots, 0}_{e+1 \text { zeros }})^{\frown}(1)^{\frown} b .
$$

A pair $(a, c)$ in $\mathscr{C}^{2}$ is a (I, $\left.n\right)$-code, if $a$ is a $(\mathrm{I}, n)$-precode and $c \in\left[T_{a}\right]$. If $(a, c)$ is a (I, $n)$-code, let $H_{n}^{\mathrm{I}}(a, c)$ be the image of $c$ under the canonical homeomorphism from $\left[T_{a}\right]$ onto $^{\omega} 2$.

Given $\left(a_{0}^{\mathrm{I}}, c_{0}^{\mathrm{I}}\right) \in \mathscr{C}^{2}$, define a (possibly finite) sequence $a_{0}^{\mathrm{I}}, c_{0}^{\mathrm{I}}, d_{0}^{\mathrm{I}}, a_{1}^{\mathrm{I}}, c_{1}^{\mathrm{I}}, d_{1}^{\mathrm{I}}, a_{2}^{\mathrm{I}}$, $c_{2}^{\mathrm{I}}, d_{2}^{\mathrm{I}}, \ldots$ by

4.1. $\left\langle d_{i}^{\mathrm{I}}, a_{i+1}^{\mathrm{I}}, c_{i+1}^{\mathrm{I}}\right\rangle= \begin{cases}H_{i}^{\mathrm{I}}\left(a_{i}^{\mathrm{I}}, c_{i}^{\mathrm{I}}\right), & \text { if }\left(a_{i}^{\mathrm{I}}, c_{i}^{\mathrm{I}}\right) \text { is a }(\mathrm{I}, i) \text {-code, } \\ \text { undefined, } & \text { otherwise }\end{cases}$ 
where \langle\rangle is a recursive bijection from $\mathscr{C}^{3}$ onto $\mathscr{C}$. Notice that:

4.2. If $a_{0}^{\mathrm{I}}, a_{1}^{\mathrm{I}}, a_{2}^{\mathrm{I}}, \ldots, a_{n}^{\mathrm{I}}, d_{0}^{\mathrm{I}}, d_{1}^{\mathrm{I}}, d_{2}^{\mathrm{I}}, \ldots, d_{n-1}^{\mathrm{I}}$, and $c_{n}^{\mathrm{I}}$ are all given, then there is a unique sequence $c_{0}^{\mathrm{I}}, \ldots, c_{n-1}^{\mathrm{I}}$ such that for all $i<n,\left\langle d_{i}^{\mathrm{I}}, a_{i+1}^{\mathrm{I}}, c_{i+1}^{\mathrm{I}}\right\rangle=H_{i}^{\mathrm{I}}\left(a_{i}^{\mathrm{I}}, c_{i}^{\mathrm{I}}\right)$.

We define (II, $n$ )-codes in an analogous manner, using the pointclass $\Gamma_{n}^{\text {II }}$ rather than $\Gamma_{n}^{\mathrm{I}}$. In this case also, a pair $\left(a_{0}^{\mathrm{II}}, c_{0}^{\mathrm{II}}\right)$ yields a (possibly finite) sequence $a_{0}^{\mathrm{II}}, c_{0}^{\mathrm{II}}$, $d_{0}^{\mathrm{II}}, a_{1}^{\mathrm{II}}, c_{1}^{\mathrm{II}}, d_{1}^{\mathrm{II}}, a_{2}^{\mathrm{II}}, c_{2}^{\mathrm{II}}, d_{2}^{\mathrm{II}}, \ldots$ as in 4.1 , and the analog of 4.2 is valid.

Now we describe the game $\mathscr{G}^{*}$. I plays $\left(a_{0}^{\mathrm{I}}, c_{0}^{\mathrm{I}}\right) \in \mathscr{C}^{2}$ and II plays $\left(a_{0}^{\mathrm{II}}, c_{0}^{\mathrm{II}}\right) \in \mathscr{C}^{2}$, both playing one integer at a time. Thus they determine sequences $a_{0}^{\mathrm{I}}, c_{0}^{\mathrm{I}}, d_{0}^{\mathrm{I}}, a_{1}^{\mathrm{I}}, c_{1}^{\mathrm{I}}$, $d_{1}^{\mathrm{I}}, \ldots$ and $a_{0}^{\mathrm{II}}, c_{0}^{\mathrm{II}}, d_{0}^{\mathrm{II}}, a_{1}^{\mathrm{II}}, c_{1}^{\mathrm{II}}, d_{1}^{\mathrm{II}}, \ldots$, as in 4.1 . If there is an $i$ such that $d_{i}^{\mathrm{I}}$ or $d_{i}^{\mathrm{II}}$ does not exist, then the first player to fail in this manner loses, that is, if $i_{0}$ is the least $i$ such that either $d_{i}^{\mathrm{I}}$ or $d_{i}^{\mathrm{II}}$ does not exist, then I wins iff $d_{i_{0}}^{\mathrm{I}}$ exists.

Assume all $d_{i}^{\mathrm{I}}$ and $d_{i}^{\mathrm{II}}$ exist. Then I and II have played as follows:

$\begin{array}{llllllllllll}\text { 4.3. } & \text { I } & d_{0}^{\mathrm{I}} & & & d_{1}^{\mathrm{I}} & & d_{2}^{\mathrm{I}} & & d_{3}^{\mathrm{I}} & & \cdots \\ & \mathrm{II} & & d_{0}^{\mathrm{II}} & & d_{1}^{\mathrm{II}} & & d_{2}^{\mathrm{II}} & & d_{3}^{\mathrm{II}} & \cdots\end{array}$

$\left(\right.$ all $d_{i}$ 's in $\left.\mathscr{C}\right)$

Let $\varphi: \mathscr{C} \rightarrow \omega$ be some trivial norm of length $\omega$ (one such that the prewellordering is $\Delta_{1}^{1}$, and for all $n \in \omega$ there is a $\Delta_{1}^{1} d \in \mathscr{C}$ such that $\varphi(d)=n$ ), and let $B: \mathscr{C} \rightarrow \mathbf{R}$ be a $\Delta_{1}^{1}$ bijection. Let $y(i)=\varphi\left(d_{i}^{\mathrm{I}}\right)$ and let $x_{i}=B\left(d_{i}^{\mathrm{II}}\right)$. Thus, via $\varphi$ and $B$, we can view the plays of $\mathscr{G}^{*}$ in 4.3 as being the following:

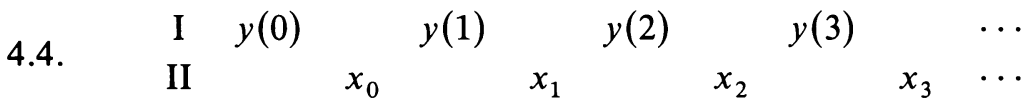

$$
\left(y(i) \in \omega, x_{i} \in \mathbf{R}\right)
$$

Notice that 4.4 is the same as 3.6 ; that is, a run of the game $\mathscr{G}^{*}$ (in which all $d_{i}$ 's exist) simulates a run of $\mathscr{G}$. I wins the run of $\mathscr{G} *$ iff I wins the corresponding run of $\mathscr{G}$. This completes the definition of $\mathscr{G}^{*}$.

This game differs from the Harringtori-Kechris simulations [3] in two ways. First, in $\mathscr{G}^{*}$ each move is coded with respect to a different pointclass $\left(\Gamma_{0}^{\mathrm{I}}, \Gamma_{0}^{\mathrm{II}}, \Gamma_{1}^{\mathrm{I}}, \Gamma_{1}^{\mathrm{II}}, \ldots\right)$, whereas in [3] all moves are coded with respect to the same pointclass. This is a technical detail which does not require any significant changes in the proof. The second difference is more important. In [3] the games being simulated were coded ordinal games, that is, the payoff set depends not on the reals played but only on the ordinals encoded by the reals. For the game $\mathscr{G}$ of this paper, this is true for I's moves; $I$ is playing integers (finite ordinals). Therefore the Harrington-Kechris proof goes through in this case, and shows that if I has a winning strategy in $\mathscr{G}^{*}$ then I has a winning strategy in $\mathscr{G}$ which is simple (details in §5). But II is not playing ordinals, and the Harrington-Kechris proof does not show that if II wins $\mathscr{G}^{*}$ then he wins $\mathscr{G}$. A different (but related) argument will be given in this case.

5. Proof of Lemma A. Let $s$ be a winning strategy for I for $\mathscr{G}^{*}$. For any $(a, c) \in \mathscr{C}^{2}, s^{*}[a, c]$ denotes the run of the game $\mathscr{G}^{*}$ in which II plays $(a, c)$ and I plays according to the strategy $s$. We now describe a strategy $\sigma$ for I for $\mathscr{G}$. 


\section{First move.}

5.1. Claim. There exists a (II,0)-precode $a_{0}^{\mathrm{II}}$ and an $m_{0} \in \omega$ such that for all $c \in\left[T_{a_{0}^{\mathrm{II}}}\right]$, in the run $s^{*}\left[a_{0}^{\mathrm{II}}, c\right]$, I plays a $d_{0}^{\mathrm{I}}$ such that $\varphi\left(d_{0}^{\mathrm{I}}\right)=m_{0}$.

Proof of Claim. Fix a $b_{0} \in \mathscr{C}$ such that $b_{0} \equiv_{T} s$. For $e \in \omega$ consider runs of $\mathscr{G}^{*}$ in which I plays by $s$ and II plays $a_{0}^{\mathrm{II}}=\left\langle e, b_{0}\right\rangle$. For any $c\left(=c_{0}^{\mathrm{II}}\right)$ played by II, I will play an $\left(a_{0}^{\mathrm{I}}, c_{0}^{\mathrm{I}}\right)$ which is a $(\mathrm{I}, 0)$-code-if not, I would lose. Hence for any $c \in C$, there is an $m^{e}(c) \in \omega$ such that $s^{*}\left[a_{0}^{\mathrm{II}}, c\right]$ has I play $d_{0}^{\mathrm{I}}$ such that $\varphi\left(d_{0}^{\mathrm{I}}\right)=$ $m^{e}(c)$. There must be a nonmeager set on which the map $c \mapsto m^{e}(c)$ is constant, hence (using the Baire property) there must exist a perfect binary tree $T \subset 2^{<\omega}$ such that $c \mapsto m^{e}(c)$ is constant on $[T]$. Let

$$
\begin{aligned}
R^{e}=\left\{z \in \mathbf{R}: z \text { encodes a perfect binary tree } T^{z}\right. \text { such that } \\
\text { the function } \left.c \mapsto m^{e}(c) \text { is constant on }\left[T^{z}\right]\right\} .
\end{aligned}
$$

(In the definition of $R^{e}$, above, encodes means via any fixed recursive scheme for encoding binary trees by reals-it does not refer to the coding of trees via (I, $n$ )-precodes or (II, $n$ )-precodes.)

The relation $R=\left\{(e, z) \in \omega \times \mathbf{R}: z \in R^{e}\right\}$ is $\Delta_{0}^{\mathrm{I}}\left(b_{0}\right)$. To see this, notice how $m^{e}(c)=\varphi\left(d_{0}^{\mathrm{I}}\right)$ is defined from $s^{*}\left[a_{0}^{\mathrm{II}}, c\right](4.1)$ and notice that in $s^{*}\left[a_{0}^{\mathrm{II}}, c\right]$ I must play a $(\mathrm{I}, 0)$-code, recall that $s$ and $a_{0}^{\text {II }}$ are recursive-in- $b_{0}$, and of course, use the closure properties of $\Delta_{0}^{\mathrm{I}}$ given in Lemma 3.1, part (5). By 3.1, part (8), $R$ has a $\Delta_{0}^{\mathrm{II}}\left(b_{0}\right)$ uniformization $e \mapsto z_{e}$. Hence (by the $S-m-n$ theorem) there is a recursive-in- $b_{0}$ function $e \mapsto \hat{e}$ such that for all $e,\left\langle\hat{e}, b_{0}\right\rangle$ is a (II, 0$)$-precode which encodes the tree $T^{z_{e}}$, that is, $T_{\left\langle\hat{e}, b_{0}\right\rangle}=T^{z_{e}}$. By the recursion theorem, there is an $e_{0}$ such that $T_{\left\langle e_{0}, b_{0}\right\rangle}=T_{\left\langle\hat{e}_{0}, b_{0}\right\rangle}$. Let $a_{0}^{\mathrm{II}}=\left\langle e_{0}, b_{0}\right\rangle$. Then $a_{0}^{\mathrm{II}}$ is a (II, 0)-precode and the function $c \mapsto m^{e_{0}}(c)$ is constant on the tree $\left[T_{a_{0}^{\mathrm{II}}}\right]$. That is, $a_{0}^{\mathrm{II}}$ satisfies 5.1 - thus the claim has been proved.

We now give I's first move in $\mathscr{G}$ according to the strategy $\sigma$.

5.2. Fix an $a_{0}^{\text {II }}$ and an $m_{0}$ satisfying Claim 5.1. Let $\sigma()=m_{0}$.

Second move. We next describe I's second move in $\mathscr{G}$ according to the strategy $\sigma$, that is, we describe a function $x_{0} \mapsto \sigma\left(x_{0}\right)$ from $\mathbf{R}$ into $\omega$; moreover, this function will be in $\Lambda$. Consider now a fixed $x_{0}$ in $\mathbf{R}$ (which, of course, is II's first move in $\mathscr{G}$ after I has played $\left.m_{0}\right)$. For any $(a, c) \in \mathscr{C}^{2}$, let $c_{0}^{\mathrm{II}}(a, c)$ be the uniquely determined $c_{0}^{\text {II }}$ of 4.2 (actually of 4.2 with superscript I's replaced by II's), where $a_{0}^{\text {II }}$ is as in 5.2, $a_{1}^{\mathrm{II}}=a, d_{0}^{\mathrm{II}}=B^{-1}\left(x_{0}\right)$, and $c_{1}^{\mathrm{II}}=c$.

5.3. Claim. There exists a (II, 1)-precode $a_{1}^{\mathrm{II}}=a_{1}^{\mathrm{II}}\left(x_{0}\right)$ and an $m_{1}=m_{1}\left(x_{0}\right) \in \omega$ such that for all $c \in\left[T_{a_{1}^{\mathrm{II}}}\right]$, if $a_{0}^{\mathrm{II}}$ is as in 5.2 and $c_{0}^{\mathrm{II}}=c_{0}^{\mathrm{II}}\left(a_{1}^{\mathrm{II}}, c\right)$, then in the run $s^{*}\left[a_{0}^{\mathrm{II}}, c_{0}^{\mathrm{II}}\right]$, I plays a $d_{1}^{\mathrm{I}}$ such that $\varphi\left(d_{1}^{\mathrm{I}}\right)=m_{1}$.

Proof of Claim. Let $b_{1}=\left\langle b_{0}, x_{0}\right\rangle$. For $e \in \omega$ consider runs of $\mathscr{G}^{*}$ in which I plays by $s$, II plays $a_{0}^{\mathrm{II}}$ of 5.2 , and II plays $c_{0}^{\mathrm{II}}=c_{0}^{\mathrm{II}}\left(a_{1}^{\mathrm{II}}, c\right)$, where $a_{1}^{\mathrm{II}}=\left\langle e, b_{1}\right\rangle$ and $c$ is an arbitrary element of $\mathscr{C}$. The proof is now similar to that of the first move. For any $c$ there is an $m_{1}^{e}(c) \in \omega$ such that in this run of $\mathscr{G}^{*}$, I plays a $d_{1}^{\mathrm{I}}$ such that $\varphi\left(d_{1}^{\mathrm{I}}\right)=m_{1}^{e}(c)$. And for any $e$, the set

$$
R^{e}=\left\{z \in \mathbf{R}: z \text { encodes a perfect binary tree } T^{z}\right. \text { such that }
$$

$$
\text { the function } \left.c \mapsto m_{1}^{e}(c) \text { is constant on }\left[T^{z}\right]\right\}
$$


is nonempty, and the relation $\left\{(e, z): z \in R^{e}\right\}$ is $\Delta_{1}^{\mathrm{I}}\left(b_{1}\right)$, hence has a $\Delta_{1}^{\mathrm{II}}\left(b_{1}\right)$ uniformization. This gives a recursive-in- $b_{1}$ function $e \mapsto \hat{e}$ such that for all $e$, $\left\langle\hat{e}, b_{1}\right\rangle$ is a (II, 1)-precode such that $T_{\left\langle\hat{e}, b_{1}\right\rangle}=T^{z_{e}}$. Again use the recursion theorem to get a fixed point $e_{1}$ such that $T_{\left\langle e_{1}, b_{1}\right\rangle}=T_{\left\langle\hat{e}_{1}, b_{1}\right\rangle}$. Then $a_{1}^{\mathrm{II}}=\left\langle e_{1}, b_{1}\right\rangle$ satisfies the claim.

Thus we have shown that for any fixed $x_{0} \in \mathbf{R}$, there exist an $a_{1}^{\mathrm{II}}$ and an $m_{1}$ such that the triple $\left(x_{0}, a_{1}^{\mathrm{II}}, m_{1}\right)$ satisfies Claim 5.3. The relation $\left\{\left(x_{0}, a_{1}^{\mathrm{II}}, m_{1}\right)\right.$ : $\left(x_{0}, a_{1}^{\mathrm{II}}, m_{1}\right)$ satisfies 5.3$\}$ is in $\Lambda$, in fact in $\Gamma_{1}^{\mathrm{II}}$. (The point is, that thus far in the proof of Lemma A, we have never even mentioned, directly or indirectly, any pointclass larger than $\Gamma_{1}^{\mathrm{II}}$. The larger classes $\Gamma_{2}^{\mathrm{I}}, \Gamma_{2}^{\mathrm{II}}, \Gamma_{3}^{\mathrm{I}}, \Gamma_{3}^{\mathrm{II}}, \ldots$ which are involved in the definition of the payoff set of $\mathscr{G}^{*}$ are not involved in the statement or proof of 5.3. They will, of course, come up in the analogous claim for moves $3,4, \ldots$ of $\mathscr{G}$. Thus this relation is in $\Lambda$.) So by definition of useful (2.2), this relation has a $\Lambda$ uniformization, that is, there exist functions $x_{0} \mapsto a_{1}^{\text {II }}\left(x_{0}\right)$ and $x_{0} \mapsto m_{1}\left(x_{0}\right)$ such that both functions are in $\Lambda$, and, for all $x_{0} \in \mathbf{R}$, the triple $\left(x_{0}, a_{1}^{\mathrm{II}}\left(x_{0}\right), m_{1}\left(x_{0}\right)\right)$ satisfies Claim 5.3. We now describe I's second move in $\mathscr{G}$ according to $\sigma$.

5.4. Fix functions $x_{0} \mapsto a_{1}^{\mathrm{II}}\left(x_{0}\right)$ and $x_{0} \mapsto m_{1}\left(x_{0}\right)$ such that both functions are in $\Lambda$ and such that for any $x_{0} \in \mathbf{R}, x_{0}, a_{1}^{\mathrm{II}}\left(x_{0}\right)$, and $m_{1}\left(x_{0}\right)$ satisfy Claim 5.3. Let $\sigma\left(x_{0}\right)=m_{1}\left(x_{0}\right)$.

Other moves. This procedure continues by induction. We thus produce a function $\sigma: \mathbf{R}^{<\omega} \rightarrow \omega$ such that for all $n \in \omega, \sigma \uparrow \mathbf{R}^{n}$ is in $\Lambda$, that is, $\sigma$ is a simple strategy for I for $\mathscr{G}$. (It remains to be proved that $\sigma$ is a winning strategy.) We also have a function from $\mathbf{R}^{<\omega}$ into $\mathscr{C}$ which assigns to each $\left(x_{0}, \ldots, x_{n-1}\right) \in \mathbf{R}^{n}$ a (II, $n$ )precode $a_{n}^{\text {II }}\left(x_{0}, \ldots, x_{n-1}\right)$ such that the following property holds:

5.5. For any $c \in\left[T_{a_{n}^{\mathrm{II}}\left(x_{0}, \ldots, x_{n-1}\right)}\right]$, if $c_{0}^{\mathrm{II}}$ is determined as in 4.2 , given $a_{0}^{\mathrm{II}}, a_{1}^{\mathrm{II}}\left(x_{0}\right), \ldots, a_{n}^{\mathrm{II}}\left(x_{0}, \ldots, x_{n-1}\right), d_{0}^{\mathrm{II}}=B^{-1}\left(x_{0}\right), d_{1}^{\mathrm{II}}=B^{-1}\left(x_{1}\right), \ldots, d_{n-1}^{\mathrm{II}}=$ $B^{-1}\left(x_{n-1}\right)$, and $c_{n}^{\mathrm{II}}=c$, then $s^{*}\left[a_{0}^{\mathrm{II}}, c_{0}^{\mathrm{II}}\right]$ has I play $d_{n}^{\mathrm{I}}$ such that $\varphi\left(d_{n}^{\mathrm{I}}\right)=$ $\sigma\left(x_{0}, \ldots, x_{n-1}\right)$.

We now prove that $\sigma$ is actually a winning strategy for $\mathscr{G}$. Consider a fixed run $R$ of the game $\mathscr{G}$ in which I plays according to $\sigma$. The moves of $R$ are:

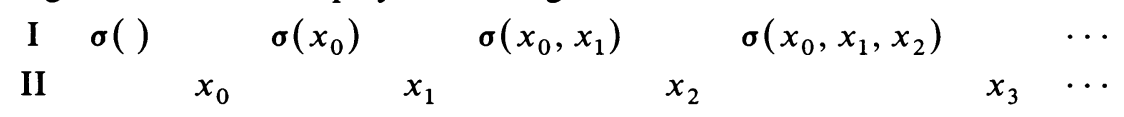

5.6. Claim. There exists a sequence $K_{0}, K_{1}, K_{2}, \ldots$ of nonempty compact sets in $\mathscr{C}$ such that $K_{0} \supset K_{1} \supset K_{2} \supset \cdots$, and such that for all $n \in \omega, K_{n}$ has the following property. For any $c \in K_{n}$, in the run $s^{*}\left[a_{0}^{\mathrm{II}}, c\right]$ of $\mathscr{G}^{*}$ :

(a) For all $i<n, d_{i}^{\mathrm{II}}$ exists, and for all $i \leqslant n, d_{i}^{\mathrm{I}}$ exists.

(b) For all $i<n, B\left(d_{i}^{\mathrm{II}}\right)=x_{i}$.

(c) For all $i \leqslant n, \varphi\left(d_{i}^{\mathrm{I}}\right)=\sigma\left(x_{0}, x_{1}, \ldots, x_{i-1}\right)$.

Assuming the claim, we show that I wins $R$. By compactness, $\bigcap_{n \in \omega} K_{n}$ is not empty. Let $c_{0}^{\mathrm{II}} \in \bigcap_{n \in \omega} K_{n}$. Consider the run $s^{*}\left[a_{0}^{\mathrm{II}}, c_{0}^{\mathrm{II}}\right]$ of $\mathscr{G}^{*}-$ call this run $R^{*}$. Now $R^{*}$ satisfies (a)-(c) of Claim 5.6 for all $n$. This means that the run $R$ of $\mathscr{G}$ and the run of $\mathscr{G}$ simulated by the run $R^{*}$ of $\mathscr{G}^{*}$ have the same outcome. Since $s$ is a winning strategy, I wins $R^{*}$, hence I also wins $R$. So all that remains to be proved is Claim 5.6. 
Let $K_{0}=\left[T_{a_{0}^{\text {II }}}\right]$. Clearly $K_{0}$ is nonempty and compact. By 5.1 and 5.2, for all $c$ in $\left[T_{a_{0}^{\mathrm{II}}}\right]$, in the run $s^{*}\left[a_{0}^{\mathrm{II}}, c\right]$, I plays a $d_{0}^{\mathrm{I}}$ such that $\varphi\left(d_{0}^{\mathrm{I}}\right)=\sigma()$. That is, $K_{0}$ satisfies the claim.

Let $J_{1}=\left[T_{a_{1}^{\mathrm{II}}\left(x_{0}\right)}\right]$. For any $c \in \mathscr{C}$ let $c_{0}^{\mathrm{II}}(c)$ be the uniquely determined $c_{0}^{\mathrm{II}}$ of 4.2 , given $a_{0}^{\mathrm{II}}, a_{1}^{\mathrm{II}}\left(x_{0}\right), d_{0}^{\mathrm{II}}=B^{-1}\left(x_{0}\right)$, and $c_{1}^{\mathrm{II}}=c$. Let $K_{1}=\left\{c_{0}^{\mathrm{II}}(c): c \in J_{1}\right\}$. A review of the construction of $c_{0}^{\mathrm{II}}(c)$ (in 4.1 and 4.2) should convince the reader of two facts:

(1) For any $c \in \mathscr{C}, c_{0}^{\mathrm{II}}(c)$ exists and is in $\left[T_{a_{0}^{\mathrm{II}}}\right]$.

(2) The function $f: c \mapsto c_{0}^{\mathrm{II}}(c)$ is continuous.

Then (1) implies that $K_{1}$ is a nonempty subset of $K_{0}$, and (2) implies that $K_{1}$ is the image of a compact set under a continuous function, hence compact. By 5.3 and 5.4, for all $c$ in $\left[T_{a_{1}^{\mathrm{II}}\left(x_{0}\right)}\right]$, in the run $s^{*}\left[a_{0}^{\mathrm{II}}, c_{0}^{\mathrm{II}}(c)\right]$, I plays a $d_{1}^{\mathrm{I}}$ such that $\varphi\left(d_{1}^{\mathrm{I}}\right)=\sigma\left(x_{0}\right)$. That is, for all $c^{\prime} \in K_{1}$, in the run $s^{*}\left[a_{0}^{\text {II }}, c^{\prime}\right]$ of $\mathscr{G}^{*}$, I plays a $d_{1}^{\mathrm{I}}$ such that $\varphi\left(d_{1}^{\mathrm{I}}\right)=\sigma\left(x_{0}\right)$, i.e., $d_{1}^{\mathrm{I}}$ satisfies (a) and (c) of the claim. Since $K_{1} \subset K_{0}$, for all $c^{\prime} \in K_{1}, d_{0}^{\mathrm{I}}$ satisfies (a) and (c) of the claim. $K_{1}$ was constructed so that $c^{\prime}=c_{0}^{\mathrm{II}}(c)$ $\in K_{1}$ was determined (via 4.2) by $d_{0}^{\text {II }}=B^{-1}\left(x_{0}\right)$; that is, for all $c^{\prime} \in K_{1}, d_{0}^{\text {II }}$ satisfies (a) and (b) of the claim. Therefore, $K_{1}$ satisfies Claim 5.6.

The rest of the sequences $K_{2}, K_{3}, \ldots$ is now constructed by induction (using 5.5). The general procedure for $K_{n}$ is the same as the procedure for $K_{1}$ described above.

6. Proof of Lemma B. The proof of this lemma is a variation on the proof of Lemma $\mathrm{A}$ given in $\S 5$. We will constantly be referring the reader back to $\S 5$ for details. Recall that we have fixed a coding of $\bar{\kappa}$-trees (by $\mathbf{T r}$ and $P^{n}$ ), and in the game $\mathscr{G}$, player II essentially builds a $\bar{\kappa}$-tree by playing reals and using this coding (see §3). Let $t$ be a winning strategy for II for $\mathscr{G}^{*}$. For any $(a, c) \in \mathscr{C}^{2},[a, c]^{*} t$ denotes the run of $\mathscr{G}^{*}$ in which I plays $(a, c)$ and II plays according to the strategy $t$. We now produce a strategy $\tau$ for II for $\mathscr{G}$. We also (as in Lemma A) produce a function from $\omega^{<\omega}$ into $\mathscr{C}$ which assigns to each $\left(m_{0}, \ldots, m_{n-1}\right) \in \omega^{n}$ a (I, $\left.n\right)$ precode $a_{n}^{\mathrm{I}}\left(m_{0}, \ldots, m_{n-1}\right)$.

6.1. Fix a (I,0)-precode $a_{0}^{\mathrm{I}}=a_{0}^{\mathrm{I}}(\mathrm{)})$ such that $a_{0}^{\mathrm{I}}$ is recursive.

First move. We must describe Il's first move in $\mathscr{G}$ according to $\tau$; that is, we must describe a function $m_{0} \mapsto \tau\left(m_{0}\right)$ from $\omega$ into $\mathbf{R}$. Consider now a fixed $m_{0}$ in $\omega$ (which, of course, is I's first move in $\mathscr{G}$ ). Fix also a $d_{0}^{\mathrm{I}} \in \mathscr{C}$ such that $d_{0}^{\mathrm{I}}$ is $\Delta_{1}^{1}$ and such that $\varphi\left(d_{0}^{\mathrm{I}}\right)=m_{0}$. For any $(a, c) \in \mathscr{C}^{2}$, let $c_{0}^{\mathrm{I}}(a, c)$ be the uniquely determined $c_{0}^{\mathrm{I}}$ of 4.2 , where $a_{0}^{\mathrm{I}}$ is as in $6.1, a_{1}^{\mathrm{I}}=a, d_{0}^{\mathrm{I}}$ is as above, and $c_{1}^{\mathrm{I}}=c$.

6.2. For any $(a, c)$ in $\mathscr{C}^{2}$, let $d_{0}^{\mathrm{II}}(a, c)$ be the $d_{0}^{\mathrm{II}} \in \mathscr{C}$ played by II in the run $\left[a_{0}^{\mathrm{I}}, c_{0}^{\mathrm{I}}(a, c)\right]^{*} t$. Notice that $d_{0}^{\mathrm{II}}(a, c)$ exists for any $a, c$-if not, II would lose the game. This $d_{0}^{\mathrm{II}}(a, c)$ encodes a subset of $\kappa_{0}, P^{0}\left(B\left(d_{0}^{\mathrm{II}}(a, c)\right)\right)$, which we now denote by $\overline{d_{0}^{\text {II }}(a, c) \text {. }}$

In the proof of Lemma $A$, as in Harrington-Kechris [3], the key fact used in constructing $\sigma$ from $s$ is the following: For any $a \in \mathscr{C}$, let $f_{a}: \mathscr{C} \rightarrow \omega$ be the function $f_{a}(c)=$ the $m_{0}$ produced in the run $s^{*}[a, c]$. Then for any $a \in \mathscr{C}$, there is a perfect binary tree $T$ such that $f_{a} \uparrow[T]$ is constant.

The analog of the above fact for Lemma B would be that for any $a \in \mathscr{C}$, there is a perfect binary tree $T$ such that the function $g_{a}: c \mapsto \overline{d_{0}^{\text {II }}(a, c)}$ from $\mathscr{C}$ to $\operatorname{Power}\left(\kappa_{0}\right)$ is 
constant for $c \in[T]$. If this were true, then we could proceed as in the proof of Lemma $\mathrm{A}$ and prove this lemma. But there is no reason to believe that it is true; indeed the function $g_{a}$ may well be one-to-one for every $a$. Although we cannot hope to find a tree $T$ such that $g_{a} \uparrow[T]$ is constant, we would like to get a tree $T$ for which the fact that $g_{a} \uparrow[T]$ is constant is as nearly true as possible. With this motivation we give the following definition.

A (I, 1)-precode $a_{1}^{\mathrm{I}}$ is good if:

$$
\begin{aligned}
& \left(\forall c \in\left[T_{a_{1}^{\mathrm{I}}}\right]\right)\left(\forall \xi<\kappa_{0}\right)\left(\exists p \in 2^{<\omega}\right) \\
& {\left[p \prec c \& ( \forall c ^ { \prime } ) \left[\text { if }\left(p \prec c^{\prime} \& c^{\prime} \in\left[T_{a_{1}^{\mathrm{I}}}\right]\right)\right.\right. \text { then }} \\
& \left.\left.\quad\left(\xi \in \overline{d_{0}^{\mathrm{II}}\left(a_{1}^{\mathrm{I}}, c^{\prime}\right)} \leftrightarrow \xi \in \overline{d_{0}^{\mathrm{II}}\left(a_{1}^{\mathrm{I}}, c\right)}\right)\right]\right] .
\end{aligned}
$$

(Thus being good means that while for $\xi<\kappa_{0}$ the question of whether $\xi \in \overline{d_{0}^{\mathrm{II}}\left(a_{1}^{\mathrm{I}}, c\right)}$ is by no means independent of the choice of $c$ in $\left[T_{a_{1}^{1}}\right]$, it does depend only on finitely many coordinates of $c$.)

6.3. Claim. There exists a (I, 1$)$-precode $a_{1}^{\mathrm{I}}=a_{1}^{\mathrm{I}}\left(m_{0}\right)$ which is good.

Proof of Claim. The proof is similar to the proof of the claims of Lemma A. Fix a $b \in \mathscr{C}$ such that $b \equiv_{T} t$. For $e \in \omega$ consider runs of $\mathscr{G}^{*}$ in which II plays by $t$, I plays $a_{0}^{\mathrm{I}}$ of 6.1 , and I plays $c_{0}^{\mathrm{I}}=c_{0}^{\mathrm{I}}\left(a_{1}^{\mathrm{I}}, c\right)$, where $a_{1}^{\mathrm{I}}=\langle e, b\rangle$ and $c$ is an arbitrary element of $\mathscr{C}$. Let $D^{e}(c)$ denote $\overline{d_{0}^{\mathrm{II}}\left(a_{1}^{\mathrm{I}}, c\right)}$, where $a_{1}^{\mathrm{I}}=\langle e, b\rangle$. Then $D^{e}(c) \subset \kappa_{0}$.

A perfect binary tree $T \subset 2^{<\omega}$ is $e$-good if:

$$
\begin{aligned}
& (\forall c \in[T])\left(\forall \xi<\kappa_{0}\right)\left(\exists p \in w^{<\omega}\right) \\
& \quad\left[p \prec c \&\left(\forall c^{\prime}\right)\left[\text { if }\left(p \prec c^{\prime} \& c^{\prime} \in[T]\right) \operatorname{then}\left(\xi \in D^{e}\left(c^{\prime}\right) \leftrightarrow \xi \in D^{e}(c)\right)\right]\right] .
\end{aligned}
$$

Claim. For all $e \in \omega$ there exists an $e$-good tree.

This claim is proved by a routine Baire-category argument (or equivalently, a forcing argument). The tree is a tree of "generic" $c$ 's, the $p$ is the condition that forces $\xi$ in or out. The proof requires the fact that a well-ordered union of meager sets is meager, which is a consequence of $\mathrm{AD}$ (see [4, Proposition 1.5.1]).

Let $R$ be the binary relation $\left\{(e, z) \in \omega \times \mathbf{R}: z\right.$ encodes an $e$-good tree $\left.T^{z}\right\}$ (where encodes means via a recursive coding scheme, not via (I, $n$ )-codes). Then by parts (5) and (7) of Lemma 3.1, $R$ is $\Delta_{0}^{\mathrm{II}}(b)$ (see also 4.1 and 6.2 for the definition of how $D^{e}(c)$ is constructed from $\left[a_{0}^{\mathrm{I}}, c_{0}^{\mathrm{I}}\right]^{*} t$, and see 3.4). Hence by part 9 of Lemma 3.1, $R$ has a $\Delta_{1}^{\mathrm{I}}(b)$ uniformization $e \mapsto z_{e}$. Now, as in the proof of Lemma A, there is a recursive-in- $b$ function $e \mapsto \hat{e}$ such that for all $e,\langle\hat{e}, b\rangle$ is a (I,1)-precode and the tree $T_{\langle\hat{e}, b\rangle}$ is e-good. By the recursion theorem, there is an $e_{0}$ such that $T_{\left\langle e_{0}, b\right\rangle}=$ $T_{\left\langle\hat{e}_{0}, b\right\rangle}$, and then $a_{1}^{\mathrm{I}}=\left\langle e_{0}, b\right\rangle$ is a $(\mathrm{I}, 1)$-precode which is good. This completes the proof of Claim 6.3.

We now describe II's first move in $\mathscr{G}$ according to the strategy $\tau$.

6.4. Fix a function $m_{0} \mapsto a_{1}^{\mathrm{I}}\left(m_{0}\right)$ such that for any $m_{0} \in \omega, a_{1}^{\mathrm{I}}\left(m_{0}\right)$ satisfies Claim 6.3. In the proof of Lemma A, as in Harrington-Kechris [3], we defined $\sigma$ by having it assume that constant value determined by the (II, $n$ )-precode $a_{n}^{\text {II }}$. In this construction there is no constant value. The tree $T_{a_{1}^{\mathrm{I}}\left(m_{0}\right)}$ does not determine a unique subset of $\kappa_{0}$-every branch $c \in\left[T_{a_{1}^{\mathrm{I}}\left(m_{0}\right)}\right]$ gives us a different subset. We will define 
the strategy $\tau\left(m_{0}\right)$ to play the union of all possible subsets given by branches $c$ of the tree $T_{a_{1}^{\mathrm{I}}\left(m_{0}\right)}$. A formal statement of this follows.

Let

$$
Q_{0}\left(m_{0}\right)=\left\{\xi<\kappa_{0}:\left(\exists c \in\left[T_{a_{1}^{\mathrm{I}}\left(m_{0}\right)}\right]\right)\left(\xi \in \overline{d_{0}^{\mathrm{II}}\left(a_{1}^{\mathrm{I}}\left(m_{0}\right), c\right)}\right)\right\}
$$

Let $x_{0}\left(m_{0}\right)$ be a fixed real such that $P^{0}\left(x_{0}\left(m_{0}\right)\right)$ is $Q_{0}\left(m_{0}\right)$. (That is, $x_{0}\left(m_{0}\right)$ is a code for the set $Q_{0}\left(m_{0}\right) \subset \kappa_{0}$. We are using countable choice.) Let $\tau\left(m_{0}\right)=x_{0}\left(m_{0}\right)$.

Second move. We next define II's second move in $\mathscr{G}$ according to the strategy $\tau$. That is, we define a function $\left(m_{0}, m_{1}\right) \mapsto \tau\left(m_{0}, m_{1}\right)$ from $\omega^{2}$ into $\mathbf{R}$. Consider now a fixed $\left(m_{0}, m_{1}\right) \in \omega^{2}$, which is I's first two moves. Let $d_{0}^{\mathrm{I}}=d_{0}^{\mathrm{I}}\left(m_{0}\right)$ be the same element of $\mathscr{C}$ as in the first move, where we fixed it to be $\Delta_{1}^{1}$ and such that $\varphi\left(d_{0}^{\mathrm{I}}\right)=m_{0}$. Fix also a $d_{1}^{\mathrm{I}}=d_{1}^{\mathrm{I}}\left(m_{1}\right)$ such that $d_{1}^{\mathrm{I}}$ is $\Delta_{1}^{1}$ and such that $\varphi\left(d_{1}^{\mathrm{I}}\right)=m_{1}$. For any $(a, c)$ in $\mathscr{C}^{2}$, let $c_{0}^{\mathrm{I}}(a, c)$ and $c_{1}^{\mathrm{I}}(a, c)$ be the $c_{0}^{\mathrm{I}}$ and $c_{1}^{\mathrm{I}}$ of 4.2 , where $a_{0}^{\mathrm{I}}$ is as in 6.1, $a_{1}^{\mathrm{I}}=a_{1}^{\mathrm{I}}\left(m_{0}\right)$ (of 6.4), $a_{2}^{\mathrm{I}}=a, d_{0}^{\mathrm{I}}$ and $d_{1}^{\mathrm{I}}$ are as above, and $c_{2}^{\mathrm{I}}=c$.

6.5. For any $(a, c) \in \mathscr{C}^{2}$, let $d_{0}^{\mathrm{II}}(a, c)$ and $d_{1}^{\mathrm{II}}(a, c)$ be the $d_{0}^{\mathrm{II}}$ and $d_{1}^{\mathrm{II}}$ played by II in the run $\left[a_{0}^{\mathrm{I}}, c_{0}^{\mathrm{I}}(a, c)\right]^{*} t$. Notice that $d_{0}^{\mathrm{II}}(a, c)$ and $d_{1}^{\mathrm{II}}(a, c)$ exist for any $a, c$. (The notation $d_{0}^{\mathrm{II}}(a, c)$ is somewhat ambiguous, since we gave a different definition of $d_{0}^{\mathrm{II}}(a, c)$ in 6.2. But $d_{0}^{\mathrm{II}}(a, c)$, as defined here in 6.5 , is the same element of $\mathscr{C}$ as $d_{0}^{\mathrm{II}}\left(a_{1}^{\mathrm{I}}\left(m_{0}\right), c_{1}^{\mathrm{I}}(a, c)\right)$ under Definition 6.2. So in practice this will cause no problem; $d_{0}^{\text {II }}$ always denotes II's first play in the game simulated by a given run of $\mathscr{G}^{*}$, regardless of whether the argument of the function $d_{0}^{\mathrm{II}}(a, c)$ refers to the $(\mathrm{I}, 1)$-code or the (I, 2)-code played by $\mathrm{I}$ in this run.) $d_{0}^{\mathrm{II}}(a, c)$ and $d_{1}^{\mathrm{II}}(a, c)$ encode subsets of $\kappa_{0}$

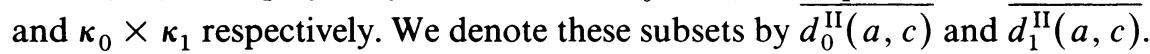

A (I, 2)-precode $a_{2}^{\mathrm{I}}$ is good if:

$$
\begin{aligned}
& \left(\forall c \in\left[T_{a_{2}}\right]\right)\left(\forall\left(\xi_{0}, \xi_{1}\right) \in \kappa_{0} \times \kappa_{1}\right)\left(\exists p \in 2^{<\omega}\right) \\
& {\left[p \prec c \& ( \forall c ^ { \prime } ) \left[\text { if }\left(p \prec c^{\prime} \& c^{\prime} \in\left[T_{a_{2}^{\mathrm{I}}}\right]\right)\right.\right. \text { then }} \\
& \left.\left.\left(\left(\xi_{0}, \xi_{1}\right) \in \overline{d_{1}^{\mathrm{II}}\left(a_{2}^{\mathrm{I}}, c^{\prime}\right)} \leftrightarrow\left(\xi_{0}, \xi_{1}\right) \in \overline{d_{1}^{\mathrm{II}}\left(a_{2}^{\mathrm{I}}, c\right)}\right)\right]\right] .
\end{aligned}
$$

Now $c_{1}^{\mathrm{I}}(a, c) \in \mathscr{C}$ is totally determined by $a$ and $c$, since we have fixed forever $a_{0}^{\mathrm{I}}$, $a_{1}^{\mathrm{I}}, d_{0}^{\mathrm{I}}$, and $d_{1}^{\mathrm{I}}$. If $T \subset 2^{<\omega}$ is a perfect binary tree, we say that the tree $T$ pins down the first coordinate of $c_{1}^{\mathrm{I}}\left(a_{2}^{\mathrm{I}}, c\right)$ if there is a $q_{0} \in 2$ such that for all $c \in[T],\left(q_{0}\right)$ is an initial segment of $c_{1}^{\mathrm{I}}\left(a_{2}^{\mathrm{I}}, c\right)$.

6.6. Claim. There exists a (I, 2)-precode $a_{2}^{\mathrm{I}}=a_{2}^{\mathrm{I}}\left(m_{0}, m_{1}\right)$ such that $a_{2}^{\mathrm{I}}$ is good and such that the tree $T_{a_{2}^{\mathrm{I}}}$ pins down the first coordinate of $c_{1}^{\mathrm{I}}\left(a_{2}^{\mathrm{I}}, c\right)$.

The proof of this claim is similar to the proof of Claim 6.3. For every $e \in \omega$ we consider runs of $\mathscr{G}^{*}$ in which II plays by $t$, I plays $a_{0}^{\mathrm{I}}$ and $c_{0}^{\mathrm{I}}=c_{0}^{\mathrm{I}}\left(a_{2}^{\mathrm{I}}, c\right)$, where $a_{2}^{\mathrm{I}}=\langle e, b\rangle$ and $c$ is arbitrary. We get a recursive-in- $b$ function $e \mapsto \hat{\mathrm{e}}$ such that for any $e,\langle\hat{e}, b\rangle$ is a $(\mathrm{I}, 2)$-precode such that the tree $T_{\langle\hat{e}, b\rangle}$ is $e$-good and pins down the first coordinate of $c_{1}^{\mathrm{I}}\left(a_{2}^{\mathrm{I}}, c\right)$. Then the recursion theorem gives an $e_{1}$ such that $T_{\left\langle e_{1}, b\right\rangle}=T_{\left\langle\hat{e}_{1}, b\right\rangle}$, and so $a_{2}^{\mathrm{I}}=\left\langle e_{1}, b\right\rangle$ satisfies the claim. Details are left to the reader.

We now describe II's second move in $\mathscr{G}$ according to the strategy $\tau$. 
6.7. Fix a function $\left(m_{0}, m_{1}\right) \mapsto a_{2}^{\mathrm{I}}\left(m_{0}, m_{1}\right)$ such that for any $\left(m_{0}, m_{1}\right) \in \omega^{2}$, $a_{2}^{\mathrm{I}}\left(m_{0}, m_{1}\right)$ satisfies Claim 6.6.

Let

$$
\begin{aligned}
Q_{1}\left(m_{0}, m_{1}\right)=\left\{\left(\xi_{0}, \xi_{1}\right)\right. & \in \kappa_{0} \times \kappa_{1}:\left(\exists c \in\left[T_{a_{2}^{\mathrm{I}}\left(m_{0}, m_{1}\right)}\right]\right) \\
{\left[\left(\xi_{0}\right)\right.} & \left.\left.\in \overline{d_{0}^{\mathrm{II}}\left(a_{2}^{\mathrm{I}}\left(m_{0}, m_{1}\right), c\right)} \&\left(\xi_{0}, \xi_{1}\right) \in \overline{d_{1}^{\mathrm{II}}\left(a_{2}^{\mathrm{I}}\left(m_{0}, m_{1}\right), c\right)}\right]\right\} .
\end{aligned}
$$

Let $x_{1}\left(m_{0}, m_{1}\right)$ be a fixed real which is a code for $Q_{1}\left(m_{0}, m_{1}\right)$. Let $\tau\left(m_{0}, m_{1}\right)=$ $x_{1}\left(m_{0}, m_{1}\right)$.

Other moves. This procedure continues by induction. We thus produce a function from $\omega^{<\omega}$ into $\mathscr{C}$ which assigns to each $\bar{m}=\left(m_{0}, \ldots, m_{n-1}\right) \in \omega^{n}$ a (I, $\left.n\right)$-precode $a_{n}^{\mathrm{I}}(\bar{m})=a_{n}^{\mathrm{I}}\left(m_{0}, \ldots, m_{n-1}\right)$, where $a_{n}^{\mathrm{I}}(\bar{m})$ satisfies two properties (goodness and pinning down coordinates) which are defined below.

6.8. For any $c \in\left[T_{a_{n}^{\mathrm{I}}(\bar{m})}\right]$, let $c_{0}^{\mathrm{I}}\left(a_{n}^{\mathrm{I}}(\bar{m}), c\right), c_{1}^{\mathrm{I}}\left(a_{n}^{\mathrm{I}}(\bar{m}), c\right), \ldots, c_{n-1}^{\mathrm{I}}\left(a_{n}^{\mathrm{I}}(\bar{m}), c\right)$ be the $c_{0}^{\mathrm{I}}, c_{1}^{\mathrm{I}}, \ldots, c_{n-1}^{\mathrm{I}}$ of 4.2 , given $a_{0}^{\mathrm{I}}=a_{0}^{\mathrm{I}}(\mathrm{)}), a_{1}^{\mathrm{I}}=a_{1}^{\mathrm{I}}\left(m_{0}\right), a_{2}^{\mathrm{I}}=a_{2}^{\mathrm{I}}\left(m_{0}, m_{1}\right), \ldots, a_{n}^{\mathrm{I}}=$ $a_{n}^{\mathrm{I}}\left(m_{0}, \ldots, m_{n-1}\right)=a_{n}^{\mathrm{I}}(\bar{m}), d_{0}^{\mathrm{I}}, \ldots, d_{n-1}^{\mathrm{I}}$ fixed $\Delta_{1}^{\mathrm{l}}$ elements of $\mathscr{C}$ such that $\varphi\left(d_{i}^{\mathrm{I}}\right)=$ $m_{i}$, and $c_{n}^{\mathrm{I}}=c$. For $c \in\left[T_{a_{n}^{\mathrm{I}}(\bar{m})}\right]$, let

$$
d_{0}^{\mathrm{II}}\left(a_{n}^{\mathrm{I}}(\bar{m}), c\right), d_{1}^{\mathrm{II}}\left(a_{n}^{\mathrm{I}}(\bar{m}), c\right), \ldots, d_{n-1}^{\mathrm{II}}\left(a_{n}^{\mathrm{I}}(\bar{m}), c\right)
$$

be the $d_{0}^{\mathrm{II}}, d_{1}^{\mathrm{II}}, \ldots, d_{n-1}^{\mathrm{II}}$ played by II in the run $\left[a_{0}^{\mathrm{I}}, c_{0}^{\mathrm{I}}\left(a_{n}^{\mathrm{I}}(\bar{m}), c\right)\right]^{*} t$ of $\mathscr{G}^{*}$, and let $\overline{d_{i}^{\mathrm{II}}\left(a_{n}^{\mathrm{I}}(\bar{m}), c\right)}$ be the subset of $\left(\kappa_{0} \times \cdots \times \kappa_{i}\right)$ encoded by $d_{i}^{\mathrm{II}}\left(a_{n}^{\mathrm{I}}(\bar{m}), c\right)$. (These definitions parallel those given prior to Claim 6.6 in the construction of the second move.)

Then the function $\left(m_{0}, \ldots, m_{n-1}\right) \mapsto a_{n}^{\mathrm{I}}\left(m_{0}, \ldots, m_{n-1}\right)$ satisfies the following two properties:

6.9. (1) The precodes are good. That is, for all $\bar{m}=\left(m_{0}, \ldots, m_{n-1}\right) \in \omega^{<\omega}$ :

$$
\begin{aligned}
& \left(\forall c \in\left[T_{a_{n}^{\mathrm{I}}(\bar{m})}\right]\right)\left(\forall \bar{\xi} \in\left(\kappa_{0} \times \cdots \times \kappa_{n-1}\right)\right)\left(\exists p \in 2^{<\omega}\right) \\
& {\left[p \prec c \& ( \forall c ^ { \prime } ) \left[\text { if }\left(p \prec c^{\prime} \& c^{\prime} \in\left[T_{a_{n}^{\mathrm{I}}(\bar{m})}\right]\right)\right.\right.} \\
& \text { then } \left.\left.\left(\bar{\xi} \in \overline{d_{n-1}^{\mathrm{II}}\left(a_{n}^{\mathrm{I}}(\bar{m}), c^{\prime}\right)} \leftrightarrow \bar{\xi} \in \overline{d_{n-1}^{\mathrm{II}}\left(a_{n}^{\mathrm{I}}(\bar{m}), c\right)}\right)\right]\right] .
\end{aligned}
$$

(2) The tree $T_{a_{n}^{\mathrm{I}}}$ pins down the first $n-1$ coordinates of $c_{1}^{\mathrm{I}}$ through $c_{n-1}^{\mathrm{I}}$. That is, for all $n \in \omega$, for all $\bar{m}=\left(m_{0}, \ldots, m_{n-1}\right) \in \omega^{n}$ :

$$
\begin{aligned}
& (\forall j \text { such that } 1 \leqslant j \leqslant n-1)(\forall i \leqslant n-2)\left(\exists q_{i}^{j} \in 2\right) \\
& \qquad\left(\forall c \in\left[T_{a_{n}^{\mathrm{I}}(\bar{m})}\right]\right)(\forall j)\left[\left(q_{0}^{j}, q_{1}^{j}, \ldots, q_{n-2}^{j}\right) \prec c_{j}^{\mathrm{I}}\left(a_{n}^{\mathrm{I}}(\bar{m}), c\right)\right] .
\end{aligned}
$$

In addition to producing (I, $n$ )-precodes, as above, we also produce a strategy $\tau$ for II, as described below. 
6.10. Let

$$
\begin{aligned}
Q_{n-1}(\bar{m})= & Q_{n-1}\left(m_{0}, \ldots, m_{n-1}\right) \\
= & \left\{\left(\xi_{0}, \ldots, \xi_{n-1}\right) \in\left(\kappa_{0} \times \cdots \times \kappa_{n-1}\right):\left(\exists c \in\left[T_{a_{n}^{\mathrm{I}}(\bar{m})}\right]\right)\right. \\
& \quad\left[\left(\xi_{0}\right) \in \overline{d_{0}^{\mathrm{II}}\left(a_{n}^{\mathrm{I}}(\bar{m}), c\right)} \&\left(\xi_{0}, \xi_{1}\right) \in \overline{d_{1}^{\mathrm{II}}\left(a_{n}^{\mathrm{I}}(\bar{m}), c\right)}\right. \\
& \&\left(\xi_{0}, \xi_{1}, \xi_{2}\right) \in \overline{d_{2}^{\mathrm{II}}\left(a_{n}^{\mathrm{I}}(\bar{m}), c\right)} \& \cdots \& \\
& \left.\left.\left(\xi_{0}, \ldots, \xi_{n-1}\right) \in \overline{d_{n-1}^{\mathrm{II}}\left(a_{n}^{\mathrm{I}}(\bar{m}), c\right)}\right]\right\} .
\end{aligned}
$$

Let $x_{n-1}\left(m_{0}, \ldots, m_{n-1}\right)$ be a fixed real which is a code for $Q_{n-1}\left(m_{0}, \ldots, m_{n-1}\right) \subset$ $\left(\kappa_{0} \times \cdots \times \kappa_{n-1}\right)$. Let $\tau\left(m_{0}, \ldots, m_{n-1}\right)=x_{n-1}\left(m_{0}, \ldots, m_{n-1}\right)$.

This completes the definition of $\tau . \tau$ is clearly a function from $\omega^{<\omega}$ into $\mathbf{R}$, i.e. a strategy for II for $\mathscr{G}$. It remains to be shown that $\tau$ is a winning strategy. Notice that by definition of the $Q_{n}$ 's (6.10), $\tau$ always has II play a tree, that is, play so that $Q_{n}$ extends $Q_{n-1}$. So if II plays by $\tau$, he will never lose $\mathscr{G}$ for this trivial reason.

Fix a run $R$ of $\mathscr{G}$ in which II plays according to $\tau$. Say I plays $m_{0}, m_{1}, m_{2}, \ldots$ and therefore II plays (codes for) $Q_{0}=Q_{0}\left(m_{0}\right), Q_{1}=Q_{1}\left(m_{0}, m_{1}\right), Q_{2}=$ $Q_{2}\left(m_{0}, m_{1}, m_{2}\right), \ldots$, where $Q_{n} \subset \kappa_{0} \times \cdots \times \kappa_{n}$. Let $T=\bigcup_{n \in \omega} Q_{n}$ be the $\bar{\kappa}$-tree played by II in this run $R$. Thus if $y=\left(m_{0}, m_{1}, \ldots\right)$, then $R$ is as follows:

$$
\begin{aligned}
& \begin{array}{ccccccccccccc}
\text { 6.11. } & R & \text { I } & m_{0} & & m_{1} & & m_{2} & & m_{3} & & \cdots & y \\
& & \text { II } & & Q_{0} & & Q_{1} & & Q_{2} & & Q_{3} & \cdots & T
\end{array} \\
& \left(m_{i} \in \omega, Q_{i} \subset \kappa_{0} \times \cdots \times \kappa_{i}\right) .
\end{aligned}
$$

It must be shown that II wins $R$. By the same type of compactness argument that was used in the proof of Lemma A (5.6ff.), there is a $c_{0}^{\mathrm{I}} \in\left[T_{a_{0}^{\mathrm{I}}}\right]$ such that in the run $\left[a_{0}^{\mathrm{I}}, c_{0}^{\mathrm{I}}\right]^{*} t$ of $\mathscr{G}^{*}$ (call this run $R^{*}$ ), for all $n \in \omega, a_{n}^{\mathrm{I}}$ in $R^{*}$ is $a_{n}^{\mathrm{I}}\left(m_{0}, \ldots, m_{n-1}\right)$, $c_{n}^{\mathrm{I}} \in\left[T_{a_{n}^{\mathrm{I}}}\right]$, and $\varphi\left(d_{n}^{\mathrm{I}}\right)=m_{n}$. Let $Q_{i}^{*}$ be the subset of $\kappa_{0} \times \cdots \times \kappa_{i}$ produced by II in the run of $\mathscr{G}$ simulated by $R^{*}$, and let $T^{*}=\bigcup_{n \in \omega} Q_{n}^{*}$ be the $\bar{\kappa}$-tree played by II in this run. Thus the run of $\mathscr{G}$ simulated by $R^{*}$ looks like this:

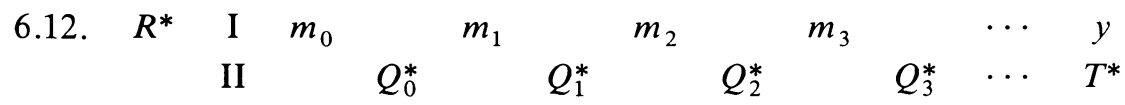

$$
\begin{aligned}
& \left(m_{i} \in \omega, Q_{i}^{*} \subset \kappa_{0} \times \cdots \times \kappa_{i}\right) .
\end{aligned}
$$

Think of $R^{*}$ as simulating an approximation to $R$. I's moves are the same: $m_{0}, m_{1}, \ldots$ II's moves are not the same. (Thus $Q_{0}^{*}, Q_{1}^{*}, \ldots$ in 6.12 are the data corresponding to $Q_{0}, Q_{1}, \ldots$ in $6.11-$ but $Q_{n}$ and $Q_{n}^{*}$ may be different subsets of $\kappa_{0} \times \cdots \times \kappa_{n}$.) But $Q_{n}^{*}$ will be an approximation to $Q_{n}$, in fact, as will be shown, an approximation from below.

Since $t$ is a winning strategy, II wins $R^{*}$. Because I's moves are the same in both 6.11 and 6.12, it clearly follows from the definition of $\mathscr{G}(3.7)$ that II wins $R$ iff either $T$ and $T^{*}$ are both well founded or $T$ and $T^{*}$ are both not well founded.

For every $n, Q_{n}^{*} \subset Q_{n}$. For simplicity of notation we verify this inclusion for the case $n=1$; the proof of the general case is similar. $Q_{1}^{*}$, is by definition, $\overline{d_{1}^{\mathrm{II}}\left(a_{2}^{\mathrm{I}}, c_{2}^{\mathrm{I}}\right)}$, 
where the function $(a, c) \mapsto d_{1}^{\mathrm{II}}(a, c)$ is as defined in 6.5, and where the $a_{2}^{\mathrm{I}}$ and $c_{2}^{\mathrm{I}}$ are those which occur in the run $R^{*}$. But $R^{*}$ was chosen so that $a_{2}^{\mathrm{I}}=a_{2}^{\mathrm{I}}\left(m_{0}, m_{1}\right)$ and $c_{2}^{\mathrm{I}} \in\left[T_{a_{2}^{\mathrm{I}}\left(m_{0}, m_{1}\right)}\right]$. Hence $Q_{1}^{*}=\overline{d_{1}^{\mathrm{II}}\left(a_{2}^{\mathrm{I}}\left(m_{0}, m_{1}\right), c_{2}^{\mathrm{I}}\right)}$. That is, $Q_{1}^{*}$ is the subset of $\kappa_{0} \times \kappa_{1}$ corresponding to one particular branch $c_{2}^{\mathrm{I}}$ of the tree $T_{a_{2}^{\mathrm{I}}\left(m_{0}, m_{1}\right)}$. But $Q_{1}=Q_{1}\left(m_{0}, m_{1}\right)$ includes, by Definition 6.7, all subsets of $\kappa_{0} \times \kappa_{1}$ corresponding to "well-behaved" branches of the tree, that is, it includes all $\overline{d_{1}^{\mathrm{II}}\left(a_{2}^{\mathrm{I}}\left(m_{0}, m_{1}\right), c\right)}$ for all "well-behaved" $c$ in $\left[T_{a_{2}^{\mathrm{I}}\left(m_{0}, m_{1}\right)}\right]$. And $c_{2}^{\mathrm{I}}$ is "well-behaved", otherwise II would lose $R^{*}$ by not playing a $Q_{1}^{*}$ which extends $Q_{0}^{*}$. So $Q_{1}^{*}$ is a subset of $Q_{1}$, as claimed. Since $Q_{n}^{*} \subset Q_{n}$, the tree $T^{*}$ is a subtree of $T$, hence if $T^{*}$ is not well founded neither is $T$. So the only way that II could possibly lose $R$ is if $T$ is not well founded but $T^{*}$ is well founded. We show that this is not the case.

Assume, towards a contradiction, that it is the case. Let $Z=\left(\xi_{0}, \xi_{1}, \ldots\right)$ be an infinite branch through $T$. Since $T^{*}$ is well founded, there is an initial segment $\bar{\xi}=\left(\xi_{0}, \ldots, \xi_{n-1}\right)$ of $Z$ such that $\bar{\xi} \notin T^{*}$. So $\bar{\xi} \notin Q_{n-1}^{*}$. Recall that $Q_{n-1}^{*}$ is $d_{n-1}^{\text {II }}\left(a_{n}^{\mathrm{I}}, c_{n}^{\mathrm{I}}\right)$, the subset of $\kappa_{0} \times \cdots \times \kappa_{n-1}$ encoded by $d_{n-1}^{\mathrm{II}}\left(a_{n}^{\mathrm{I}}, c_{n}^{\mathrm{I}}\right)$, which is II's $n$th move in $R^{*}$. Here $a_{n}^{\mathrm{I}}$ and $c_{n}^{\mathrm{I}}$ refer to those values which occur in the run $R^{*}$. By choice of $R^{*}, a_{n}^{\mathrm{I}}=a_{n}^{\mathrm{I}}(\bar{m})$, where $\bar{m}=\left(m_{0}, \ldots, m_{n-1}\right)$ and $c_{n}^{\mathrm{I}} \in\left[T_{a_{n}^{\mathrm{I}}(\bar{m})}\right]$. Since $a_{n}^{\mathrm{I}}(\bar{m})$ is good (6.9(1)), there is a condition $p \prec c_{n}^{\mathrm{I}}$ such that for every branch $c^{\prime}$ of the tree $T_{a_{n}^{\mathrm{I}}(\bar{m})}$ which extends $p, \bar{\xi}$ is not in $\overline{d_{n-1}^{\mathrm{II}}\left(a_{n}^{\mathrm{I}}(\bar{m}), c^{\prime}\right)}$. Let $l$ be the length of $p$ (w.l.o.g. $l \geqslant n$ ) and let $\bar{m}_{l}=\left(m_{0}, \ldots, m_{l}\right)$. By property (2) of 6.9, the tree $T_{a_{l+1}^{\mathrm{I}}\left(\bar{m}_{l}\right)}$ pins down the first $l$ coordinates of $c_{n}^{I}$. Since $R^{*}$ gives a branch $c_{l+1}^{\mathrm{I}}$ through the tree $T_{a_{l+1}^{\mathrm{I}}\left(\bar{m}_{l}\right)}$, these first $l$ coordinates must be those that occur in $R^{*}$, that is, they must be $p$. Therefore, for any $c^{\prime \prime} \in\left[T_{a_{l+1}^{\mathrm{I}}\left(\bar{m}_{l}\right)}\right]$, the set $\overline{d_{n-1}^{\mathrm{II}}\left(a_{l+1}^{\mathrm{I}}\left(\bar{m}_{l}\right), c^{\prime \prime}\right)} \subset \kappa_{0} \times \cdots \times$ $\kappa_{n-1}$ (defined in 6.8) does not contain $\bar{\xi}$. Hence by definition of $Q_{l}=Q_{l}\left(\bar{m}_{l}\right)$ (6.10), no extension of $\bar{\xi}$ is in $Q_{l}$. In particular, no initial segment of $Z$ is in $Q_{l}$, so $Z$ is not a branch of $T$, a contradiction.

The referee has observed that there is a way to modify the proof of 3.10 (and also the proofs in Harrington-Kechris [3]) in order to make the proof less recursion theoretic. This modification has the advantage of eliminating the recursion theorem and the uniformities from the proof. It has the disadvantage of making the definition of the game $\mathscr{G}^{*}$ of $\$ 4$ more complicated, by essentially embedding part of the proof into the definition. While we have decided not to rewrite the proof in this paper, we will pass on the referee's modification to the interested reader. We explain it below in enough detail that the reader who knows the proof of 3.10 given above will be able to follow it.

Suppose we have some method, $\mathscr{M}$, for encoding perfect trees by reals, and that this method has the same definability properties as the coding given in $\S 4$, e.g. the coding of trees via $(\mathrm{II}, 0)_{\mathscr{M}}$-precodes is $\Gamma_{0}^{\mathrm{II}}$. Then we can define the game $\mathscr{G}_{\mathcal{M}}^{*}$ in the same way that $\mathscr{G}^{*}$ was defined in $\$ 4$, except that we code trees via $\mathscr{M}$. One can formulate the properties of $\mathscr{M}$ needed to make the analogs of Lemmas A and B go through for $\mathscr{G}_{\mathcal{M}}^{*}$. For example, the following property suffices for proving the analog of Claim 5.1 for $(\mathrm{II}, 0)_{\mathscr{K}}$-precodes:

6.13. For any $\Delta_{0}^{\mathrm{I}}$ function $f: \mathbf{R}^{2} \rightarrow \mathbf{R}$, there exists a $(\mathrm{II}, 0)_{\mathscr{M}}$-precode $a_{0}^{\mathrm{II}}$ (which encodes a tree $\left.T_{a_{0}^{\mathrm{HI}}}^{\mathrm{HI}} \subset 2^{<\omega}\right)$ and an $m_{0} \in \omega$ such that for all $c \in\left[T_{a_{0}^{\mathrm{II}}}^{\mu}\right], \varphi\left(f\left(a_{0}^{\mathrm{II}}, c\right)\right)=$ $m_{0}$. 
We leave it to the reader to formulate the properties of (II, $n+1)_{\mathscr{M}}$-precodes and $(\mathrm{I}, n)_{\mathscr{M}}$-precodes. Call the coding method $\mathscr{M}$ desirable if it satisfies all of these properties. One must first prove that there exists a desirable $\mathscr{M}$. Assuming this is so, fix such an $\mathscr{M}$, consider the game $\mathscr{G}_{\mathscr{M}}^{*}$, and prove the analogs of Lemmas A and B for $\mathscr{G}_{\mathscr{M}}^{*}$ by the methods of $\$ \S 5$ and 6.

It remains to be shown that a desirable $\mathscr{M}$ exists. Of course we proved that in $\S \S 5$ and 6 for the $\mathscr{M}$ defined in $\S 4$. The referee's point is that this fact has a "classical" proof. We prove the existence of (II, 0$)_{\mathscr{M}}$-precodes which give a $\Gamma_{0}^{\mathrm{II}}$-coding of trees and satisfy 6.13 ; the rest of the proof of the existence of a desirable $\mathscr{M}$ is left to the reader. Fix a $\Gamma_{0}^{\mathrm{I}}$-coding of all $\Delta_{0}^{\mathrm{I}}$-functions from $\mathbf{R}^{2}$ into $\mathbf{R}$ by reals; let $f_{a}$ denote the function encoded by the real $a$, and let $F \subset \mathbf{R}$ be the set of codes. For any $a \in F$, let

$$
\begin{array}{r}
R^{a}=\left\{z \in \mathbf{R}: z \text { encodes a perfect binary tree } T^{z}\right. \text { such that } \\
\text { the function } \left.c \mapsto \phi\left(f_{a}(a, c)\right) \text { is constant on }\left[T^{z}\right]\right\} .
\end{array}
$$

For any $a \in F, R^{a}$ is nonempty, by the proof of 5.1. Let $R=\{(a, z): a \in F \& z \in$ $\left.R^{a}\right\}$. $R$ is $\Gamma_{0}^{\mathrm{I}}$, so $R$ has a $\Delta_{0}^{\mathrm{II}}$ uniformization. Now let $F$ be the set of $(\mathrm{II}, 0)_{\mathscr{M}}$-precodes, and let $a \in F$ encode the tree given by the above $\Delta_{0}^{\mathrm{II}}$ uniformization. This works.

7. On the ordinal $\delta_{1}^{1}(A)$.

7.1. Definition. Let $A \subset \mathbf{R}$ and let $\Lambda=\{B: w(B)<w(A)\}$. $A$ is suitable if:

(a) $w(A)$ is a limit ordinal and $\operatorname{cof}(w(A))=\omega$.

(b) For all $B \in \Lambda, \Pi_{1}^{1}(B) \subset \Lambda$.

Clearly all useful sets are suitable. In this section we study the ordinal $\delta_{1}^{1}(A)$ for suitable $A$. (We are unable to prove anything more about useful sets than about arbitrary suitable sets.) This topic has been studied in Kechris-Solovay-Steel [6]. (A $\Lambda$ satisfying 7.1(b) is called strongly-closed in [6], and the pointclasses $\Pi_{1}^{1}(A)$ for suitable $A$ are exactly what is known in [6] as the pointclass $\Gamma_{1}$ in a projective-like hierarchy of type I.) Henceforth the letter $A$ will always denote a suitable set and $\Lambda$ will always denote $\{B: w(B)<w(A)\}$. In [6, Theorem 5.1], it was shown that $\Pi_{1}^{1}(A)$ has the prewellordering property; hence by $[12,4 \mathrm{C} .14], \delta_{1}^{1}(A)$ is the length of a $\Pi_{1}^{1}(A)$-norm on a complete $\Pi_{1}^{1}(A)$ set. We give some other characterizations below.

Let $\Sigma_{0}^{1}(A)$ be the pointclass consisting of all countable unions of $\Lambda$ sets. Then $\Sigma_{0}^{1}(A)$ is $\mathbf{R}$-parametrized and is closed under \&, $\vee, \exists^{\omega}, \exists^{\mathbf{R}}$ and continuous substitution, but is not closed under $\forall^{\omega}$, and $\Pi_{1}^{1}(A)$ is $\forall^{\mathbf{R}}\left(\Sigma_{0}^{1}(A)\right)$, i.e. is the pointclass $\left\{\forall^{\mathbf{R}} B: B \in \Sigma_{0}^{1}(A)\right\}$ (cf. [6]). Let $\Pi_{0}^{1}(A)$ be the dual class of $\Sigma_{0}^{1}(A)$, that is, countable intersections of $\Lambda$ sets.

By a coding of $\kappa$ we mean a set of reals $C$ and a surjection $\varphi: C \rightarrow \kappa . C$ is the set of codes and $x \in C$ encodes the ordinal $\varphi(x)$. If $\Gamma$ is a pointclass, the coding $(C, \varphi)$ of $\kappa$ is $\Gamma$-bounded if for any set $P$ in $\Gamma$, if $P \subset C$ then $\sup \{\varphi(x): x \in P\}<\kappa$. (For example, the usual coding of $\omega_{1}$ is $\Sigma_{1}^{1}$-bounded.)

If $\Gamma$ is a parametrized pointclass, let $w(\Gamma)$ denote the ordinal $w(B)$, for $B$ a complete $\Gamma$ set.

7.2. Definition. Let $\kappa$ be a regular cardinal and let $\mathscr{P}$ be an initial segment of the Wadge degrees (that is, $\mathscr{P}$ is a pointclass closed under continuous substitution). A 
pointclass $\Gamma$ is a Steel witness for $(\mathscr{P}, \kappa)$ if:

(a) $\Gamma$ is $\mathbf{R}$-parametrized and closed under continuous substitution.

(b) $\Gamma$ and $\check{\Gamma}$ are both closed under intersection with sets in $\mathscr{P}$, that is, if $C \in \mathscr{P}$, $D \in \Gamma$ then $C \cap D \in \Gamma$, and similarly for $\breve{\Gamma}$.

(c) $w(\Gamma)$ is a limit ordinal and $\operatorname{cof}(w(\Gamma))=\kappa$.

The pair $(\mathscr{P}, \kappa)$ has the Steel property if there exists a Steel witness for $(\mathscr{P}, \kappa)$.

This concept is motivated by the following theorem, which says that for certain $(\mathscr{P}, \kappa)$ practically anything is a Steel witness.

7.3. THEOREM (STEEL [15]; AD). Let $\Gamma$ be non-self-dual such that $\exists^{\mathbf{R}} \Delta \subset \Delta$. Let $C$ be $\xi$-Suslin, where $\xi<\operatorname{cof}(w(\Gamma))$. Then for all $D \in \Gamma, C \cap D$ is in $\Gamma$.

7.4. Theorem (AD). Let $A$ be suitable and let $\Lambda=\{B: w(B)<w(A)\}$. The following four ordinals are all equal:

(a) $\delta_{1}^{1}(A)$

(b) $w\left(\Sigma_{0}^{1}(A)\right)$;

(c) $\kappa_{1}(A)=$ the least ordinal $\kappa$ such that there is a $\Lambda$-bounded coding of $\kappa$;

(d) $\kappa_{2}(A)=$ the least regular cardinal $\kappa$ such that $(\Lambda, \kappa)$ has the Steel property.

Proof. We prove the four inequalities:

$$
\kappa_{1}(A) \leqslant \kappa_{2}(A) \leqslant w\left(\Sigma_{0}^{1}(A)\right) \leqslant \delta_{1}^{1}(A) \leqslant \kappa_{1}(A) .
$$

(i) $\kappa_{1}(A) \leqslant \kappa_{2}(A)$. It will suffice to show that there is a $\Lambda$-bounded coding of $\kappa_{2}(A)$. Let $\Gamma$ be a Steel witness for $\left(\Lambda, \kappa_{2}(A)\right)$ and let $B$ be a complete $\Gamma$ set. Let $f$ : $\kappa_{2}(A) \rightarrow w(B)$ be an increasing cofinal mapping. Let

$C=\left\{x \in \mathbf{R}: x\right.$ encodes two continuous functions $g_{x}$ and $h_{x}$

$$
\left.\& g_{x}^{-1}(B) \text { is the complement of } h_{x}^{-1}(B)\right\} \text {. }
$$

Let

$$
\varphi(x)=\text { least } \xi \text { such that } f(\xi) \geqslant w\left(g_{x}^{-1}(B)\right) .
$$

Clearly $(C, \varphi)$ is a coding of $\kappa_{2}(A)$. Suppose it is not $\Lambda$-bounded-say $P$ is an unbounded set of codes, $P \in \Lambda$. Let

$$
Q(x, y) \Leftrightarrow\left[x \in P \& y \in g_{x}^{-1}(B)\right] \Leftrightarrow\left[x \in P \& y \in h_{x}^{-1}(\neg B)\right] .
$$

By definition of Steel witness (7.2), $\Gamma$ and $\breve{\Gamma}$ are closed under intersection with $\Lambda$ sets; so $Q$ is both $\Gamma$ and $\check{\Gamma}$, hence $Q$ is $\Delta$. But this contradicts the fact that every $\Delta$ set is Wadge-reducible to $Q$. To Wadge-reduce the $\Delta$ set $D$ to $Q$, fix $x_{0} \in P$ such that $f\left(\varphi\left(x_{0}\right)\right)>w(D)$, which is possible since $P$ is unbounded, and then reduce $D$ to $\left\{\left(x_{0}, y\right): y \in g_{x_{0}}^{-1}(B)\right\}$.

(ii) $\kappa_{2}(A) \leqslant w\left(\Sigma_{0}^{1}(A)\right)$. It follows from the closure properties of $\Sigma_{0}^{1}(A)$, that $w\left(\Sigma_{0}^{1}(A)\right)$ is a limit ordinal and $\Sigma_{0}^{1}(A)$ is a Steel witness for $(\Lambda, \eta)$, where $\eta=\operatorname{cof}\left(w\left(\Sigma_{0}^{1}(A)\right)\right)$. So $(\Lambda, \eta)$ has the Steel property, hence $\kappa_{2}(A) \leqslant \eta \leqslant w\left(\Sigma_{0}^{1}(A)\right)$.

(iii) $w\left(\Sigma_{0}^{1}(A)\right) \leqslant \delta_{1}^{1}(A)$. Let $B$ be a set which is both $\Sigma_{0}^{1}(A)$ and $\Pi_{0}^{1}(A)$. We show that $w(B)<\delta_{1}^{1}(A)$. Let every real $x$ encode a continuous function $f_{x}$ (in some fairly decent way), and for $x, y \in \mathbf{R}$, define

$$
\chi \triangleleft y \Leftrightarrow w\left(f_{x}^{-1}(B)\right)<w\left(f_{y}^{-1}(B)\right) .
$$


Clearly $\triangleleft$ is a well-founded relation of $\operatorname{rank} w(B)$. It is easy to see that $\triangleleft$ is $\Delta_{1}^{1}(A)$, so it has rank less than $\delta_{1}^{1}(A)$.

(iv) $\delta_{1}^{1}(A) \leqslant \kappa_{1}(A)$. Define $\sigma\left(=\sigma_{0}^{1}(A)\right)$ to be the ordinal

$\sup \left\{\operatorname{rank}(\prec): \prec\right.$ a strict well-founded relation on $\left.\mathbf{R} \& \prec \in \Sigma_{0}^{1}(A)\right\}$.

To prove (iv), we prove the following two inequalities: $\sigma \leqslant \kappa_{1}(A)$ and $\delta_{1}^{1}(A) \leqslant \sigma$.

$\sigma \leqslant \kappa_{1}(A)$. Assume, towards a contradiction, that $\kappa_{1}(A)<\sigma$. Let $\triangleleft$ be a $\Sigma_{0}^{1}(A)$ (strict) well-founded relation of rank $\kappa_{1}(A)$. Let $(C, \varphi)$ be a $\Lambda$-bounded coding of $\kappa_{1}(A)$. It is obvious that $\kappa_{1}(A)$ cannot have cofinality $\omega$, so the coding $(C, \varphi)$ must in fact be $\Sigma_{0}^{1}(A)$-bounded. Let $f: \kappa_{1}(A) \rightarrow \operatorname{Power}(\mathbf{R})$ be

$$
y \in f(\xi) \Leftrightarrow(y \in C \& \varphi(y)=\xi) .
$$

The Moschovakis coding lemma is applicable to the pointclass $\Sigma_{0}^{1}(A)$ (cf. Moschovakis [12, 7D.5] - note that this proof does not require the pointclass to be closed under $\left.\forall^{\omega}\right)$. We apply the coding lemma to the $\Sigma_{0}^{1}(A)$ well-founded relation $\triangleleft$, and thus get a $\Sigma_{0}^{1}(A)$ choice set $F$ for the function $f$; that is, $F \subset \mathbf{R}^{2}$ is $\Sigma_{0}^{1}(A)$, and:

(a) $(x, y) \in F \Rightarrow\left[x \in \operatorname{field}(\triangleleft) \& y \in f\left(\operatorname{rank}_{\triangleleft}(x)\right)\right]$.

(b) $\left(\forall \xi<\kappa_{1}(A)\right)(\exists x, y)\left(x \in \operatorname{field}(\triangleleft) \& \operatorname{rank}_{\triangleleft}(x)=\xi \&(x, y) \in F\right)$.

Then the set $P=\{y: \exists x F(x, y)\}$ is a $\Sigma_{0}^{1}(A)$ subset of $C$ and $\{\varphi(y): y \in P\}$ is $\kappa_{1}(A)$. But this contradicts the fact that the coding $(C, \varphi)$ is $\Sigma_{0}^{1}(A)$-bounded.

$\delta_{1}^{1}(A) \leqslant \boldsymbol{\sigma}$. Let $W \subset \mathbf{R}^{2}$ be an arbitrary $\Sigma_{1}^{1}(A)$ well-founded relation. It will suffice to show that there is a $\Sigma_{0}^{1}(A)$ well-founded relation on $\mathbf{R}$ whose rank is at least $\operatorname{rank}(W)$. Since $W$ is $\Sigma_{1}^{1}(A)$, there is a $V \subset \mathbf{R}^{3}$ in $\Pi_{0}^{1}(A)$ such that $W(x, y) \Leftrightarrow$ $\exists z V(x, y, z)$. Let $T$ be the following tree on $\mathbf{R}^{3}$ :

$$
\begin{aligned}
& \left\{\left(\left(x_{0}, x_{1}, z_{1}\right),\left(x_{1}, x_{2}, z_{2}\right),\left(x_{2}, x_{3}, z_{3}\right), \ldots,\left(x_{n-1}, x_{n}, z_{n}\right)\right):\right. \\
& \left.\quad V\left(x_{0}, x_{1}, z_{1}\right) \& V\left(x_{1}, x_{2}, z_{2}\right) \& V\left(x_{2}, x_{3}, z_{3}\right) \& \cdots \& V\left(x_{n-1}, x_{n}, z_{n}\right)\right\} .
\end{aligned}
$$

$T$ is well founded (since an infinite branch of $T$ gives an infinite descending sequence through $W$ ) and

$$
\operatorname{rank}(T) \geqslant \operatorname{rank}(W)
$$

(since, by induction, if $u=\left(\left(x_{0}, x_{1}, z_{1}\right), \ldots,\left(x_{n-1}, x_{n}, z_{n}\right)\right)$ is in $T$, then $\operatorname{rank}_{W}\left(x_{n}\right)$ $\leqslant \operatorname{rank}_{T}(u)$ ). Let $S_{n}$ be the set of all length $n$ sequences. Clearly for fixed $n, T \cap S_{n}$ is $\Pi_{0}^{1}(A)$, and

$$
T=\bigcap_{n \in \omega}\left[\left(T \cap S_{n}\right) \cup\left(\bigcup_{m \neq n} S_{m}\right)\right],
$$

so $T$ is $\Pi_{0}^{1}(A)$. Hence $T=\bigcap_{n \in \omega} L_{n}$, where each set $L_{n} \subset\left(\mathbf{R}^{3}\right)^{<\omega}$ is in $\Lambda$. Now define the tree $T^{\prime}$ on $\mathbf{R}^{3}$ to be

$$
\left\{\left(\bar{v}_{0}, \bar{v}_{1}, \ldots, \bar{v}_{n}\right) \in\left(\mathbf{R}^{3}\right)^{n+1}:(\forall i \leqslant n)(\forall j \leqslant n) L_{i}\left(\bar{v}_{0}, \bar{v}_{1}, \ldots, \bar{v}_{j}\right)\right\} .
$$

An infinite branch through $T^{\prime}$ gives one through $T$, hence $T^{\prime}$ must also be well founded. And $T \subset T^{\prime}$, hence $\operatorname{rank}\left(T^{\prime}\right) \geqslant \operatorname{rank}(T)$. Thus the tree $T^{\prime}$ (that is, the set of sequences in $T^{\prime}$, ordered by extension) is a well-founded relation of rank at least 
$\operatorname{rank}(W)$. Clearly for any fixed $n,\left(T^{\prime} \cap S_{n}\right)$ is in $\Lambda$, hence $T^{\prime}$ is a countable union of $\Lambda$ sets, that is, a $\Sigma_{0}^{1}(A)$ set.

By combining Theorem 7.4 with Corollary 2.4 (or Corollary 2.5), we get new local (or global) equivalences with the existence of scales. One such equivalence is particularly interesting and deserves special mention. Using the characterization of $\delta_{1}^{1}(A)$ in part (d) of 7.4 , plus 2.4 , we obtain

7.5. CoROllary (AD). Let $A$ be useful. The following are equivalent:

(a) $A$ is $w(A)$-Suslin.

(b) There is a Steel witness for $\left(\Lambda,(w(A))^{+}\right)$.

If $A$ is $w(A)$-Suslin, then by Steel's Theorem (7.3) there are lots of Steel witnesses, namely all $\Gamma$ 's such that $\operatorname{cof}(w(\Gamma))=(w(A))^{+}$and $\exists^{\mathbf{R}} \Delta \subset \Delta$. So (b) $\Rightarrow$ (a) of Corollary 7.5, above, is a converse of Steel's Theorem: if there exists even one Steel witness, then $A$ is $w(A)$-Suslin. It follows that if there is one Steel witness, then "almost every" pointclass is a Steel witness.

It is an open question whether every suitable set $A$ has the Kunen-Martin property $\delta_{1}^{1}(A)=(w(A))^{+}$. While concepts such as (b)-(d) of 7.4 may be less natural than that of $\delta_{1}^{1}(A)$, they also appear to be more promising for attacking this problem. There are many theorems about closure properties of abstract pointclasses (see Steel [15]), but no theorems about $\delta_{1}^{1}(A)$ being small (without assuming sets are scaled or $\infty$-Borel). This question is of course related to the problem of whether UNIF $\Rightarrow$ SCALES. The Kunen-Martin property for a set $A$ is absolute for all models of AD containing all reals and containing $A$, as are all the notions of 7.4 (whereas the question of whether a set $B$ admits a scale is not absolute for models of AD containing all reals and $B$ ); this is some evidence that the question of whether or not $A$ has the Kunen-Martin proeprty can be answered assuming only AD. Another approach to proving UNIF $\Rightarrow$ SCALES would be to prove that every set is effectively $\infty$-Borel, from which it would follow by Woodin's Theorem (2.6); whether a given set $B$ is effectively $\infty$-Borel is also absolute (cf. [6, Appendix B]).

We conclude by giving another equivalent of the Kunen-Martin property, one which does not involve characterizing the ordinal $\delta_{1}^{1}(A)$. Fix a suitable $A$, let $\bar{\kappa}=\kappa_{0}, \kappa_{1}, \kappa_{2}, \ldots$ be a sequence of ordinals cofinal in $w(A)$, and let $\overline{\mathscr{C}}=\left\{\mathscr{C}_{n}\right\}$ be a sequence such that each $\mathscr{C}_{n}$ is a coding scheme for $\operatorname{Power}\left(\kappa_{0} \times \cdots \times \kappa_{n}\right)$, i.e. $\mathscr{C}_{n}=\left\langle C_{n}, P^{n}\right\rangle, C_{n} \subset \mathbf{R}$, and $P^{n}: C_{n} \rightarrow \operatorname{Power}\left(\kappa_{0} \times \cdots \times \kappa_{n}\right)$ is a surjection. Any such $\overline{\mathscr{C}}$ gives us a method of encoding $\bar{\kappa}$-trees by reals (cf. 3.2 and 3.5). Let $\mathrm{WF}_{(\bar{\kappa}, \overline{\mathscr{Q}})}$ be the set of all reals that encode $\bar{\kappa}$-trees which are well founded. Call $(\bar{\kappa}, \overline{\mathscr{C}})$ reasonable if for any fixed $n$, the coding of $\operatorname{Power}\left(\kappa_{0} \times \cdots \times \kappa_{n}\right)$ is in $\Lambda$ (as in 3.4). If $(\bar{\kappa}, \overline{\mathscr{C}})$ is reasonable then it follows easily from the coding lemma that the set $\mathrm{WF}_{(\bar{\kappa}, \overline{\mathscr{C}})}$ is $\Pi_{1}^{1}(A)$. (It is also easy to see that if for some reasonable $(\bar{\kappa}, \overline{\mathscr{C}}), \mathrm{WF}_{(\bar{\kappa}, \overline{\mathscr{C}})}$ is $\Sigma_{1}^{1}(A)$, then for every reasonable $(\bar{\kappa}, \overline{\mathscr{C}}), \mathrm{WF}_{(\bar{\kappa}, \overline{\mathscr{C}})}$ is $\Sigma_{1}^{1}(A)$.)

7.6. THEOREM (AD). Let $A$ be suitable, and let $(\bar{\kappa}, \overline{\mathscr{C}})$ be reasonable. The following are equivalent:

(a) $\delta_{1}^{1}(A)=(w(A))^{+}$.

(b) $\mathrm{WF}_{(\bar{\kappa}, \overline{\mathscr{C}})}$ is not $\Sigma_{1}^{1}(A)$. 
Proof. (a) $\Rightarrow$ (b). If $\mathrm{WF}=\mathrm{WF}_{(\bar{\kappa}, \overline{\mathscr{C}})}$ is $\Sigma_{1}^{1}(A)$, then by putting together all the well-founded trees, one can produce a $\Delta_{1}^{1}(A)$ well-founded relation of rank $(w(A))^{+}$ (as in the proof of 3.9), hence $\delta_{1}^{1}(A)>(w(A))^{+}$.

(b) $\Rightarrow$ (a). WF is $\Pi_{1}^{1}(A)$, and the norm $\psi:$ WF $\rightarrow(w(A))^{+}$that maps each well-founded tree to its rank is a $\Pi_{1}^{1}(A)$-norm on WF. WF is not $\Sigma_{1}^{1}(A)$, so by Wadge's Lemma it is complete $\Pi_{1}^{1}(A)$. By [12,4C.14], $\delta_{1}^{1}(A)$ is equal to the length of a $\Pi_{1}^{1}(A)$-norm on a complete $\Pi_{1}^{1}(A)$ set, that is, to $(w(A))^{+}$.

ACKNOWLEDGEMENT. I have received many helpful comments and suggestions regarding this paper from the logicians at Caltech and UCLA, for which I am grateful. I would particularly like to thank Professor Alexander Kechris. I would also like to thank the math departments at the University of Wisconsin, where most of this research was done, and Caltech, where this paper was written.

\section{REFERENCES}

1. A. Blass, Equivalence of two strong forms of determinacy, Proc. Amer. Math. Soc. 52 (1975), 373-376.

2. C. C. Chang, Sets constructible using $L_{\kappa \kappa}$, Axiomatic Set Theory, Proc. Sympos. Pure Math., vol. 13, part I, Amer. Math. Soc., Providence, R. I., 1971, pp. 1-8.

3. L. A. Harrington and A. S. Kechris, On the determinacy of games on ordinals, Ann. Math. Logic 20 (1981), 109-154.

4. A. S. Kechris, Measure and category in effective descriptive set theory, Ann. Math. Logic 5 (1973), $337-384$

5. , Determinacy and the structure of $L(\mathbf{R})$, Proc. Conf. Recursion Theory (Cornell, 1982) (to appear).

6. A. S. Kechris, R. M. Solovay and J. R. Steel, The axiom of determinacy and the prewellordering property, Cabal Seminar 77-79, Lecture Notes in Math., vol. 839 (A. S. Kechris, D. A. Martin and Y. N. Moschovakis, eds.), Springer-Verlag, Berlin and New York, 1981, pp. 101-125.

7. K. Kunen, $\boldsymbol{A}$ model for the negation of the axiom of choice, Cambridge Summer School in Math. Logic, Lecture Notes in Math., vol. 337 (A. R. D. Mathias and H. Rogers, eds.), Springer-Verlag, Berlin and New York, 1973, pp. 489-494.

8. D. A. Martin, Y. N. Moschovakis and J. R. Steel, The extent of definable scales, Bull. Amer. Math. Soc. (N. S.) 6 (1982), 435-440.

9. Y. N. Moschovakis, Hyperanalytic predicates, Trans. Amer. Math. Soc. 129 (1967), 249-282.

10. Determinacy and prewellorderings of the continuum, Mathmatical Logic and Foundations of Set Theory (Y. Bar-Hillel, ed.), North-Holland, Amsterdam, 1970, pp. 24-62.

11. __ Uniformization in a playful universe, Bull. Amer. Math. Soc. 77 (1971), 731-736.

12. Descriptive set theory, North-Holland, Amsterdam, 1980.

13. J. Mycielski, On the axiom of determinateness, Fund. Math. 53 (1964), 205-224.

14. R. M. Solovay, The independence of DC from AD, Cabal Seminar 76-77, Lecture Notes in Math., vol. 689 (A. S. Kechris and Y. N. Moschovakis, eds.), Springer-Verlag, Berlin and New York, 1978, pp. $171-184$

15. J. R. Steel, Closure properties of pointclasses, Cabal Seminar 77-79, Lecture Notes in Math., vol. 839 (A. S. Kechris, D. A. Martin and Y. N. Moschovakis, eds.), Springer-Verlag, Berlin and New York, 1981, pp. 147-163.

16. R. Van Wesep, Wadge degrees and descriptive set theory, Cabal Seminar 76-77, Lecture Notes in Math., vol. 689 (A. S. Kechris and Y. N. Moschovakis, eds.), Springer-Verlag, Berlin and New York, 1978, pp. $151-170$.

Department of Mathematics, California institute of Technology, Pasadena, California 91125 